\title{
On Schrödinger superalgebras
}

\author{
C. DUVAL $\left({ }^{1}\right)$ and P. A. HORVÁTHY $\left({ }^{2}\right)$
}

\begin{abstract}
.
We construct, using the supersymplectic framework of Berezin, Kostant and others, two types of supersymmetric extensions of the Schrödinger algebra (itself a conformal extension of the Galilei algebra). An 'I-type' extension exists in any space dimension, and for any pair of integers $N_{+}$and $N_{-}$. It yields an $N=N_{+}+N_{-}$superalgebra, which generalizes the $N=1$ supersymmetry Gauntlett et al. found for a free spin- $\frac{1}{2}$ particle, as well as the $N=2$ supersymmetry of the fermionic oscillator found by Beckers et al. In two space dimensions, new, 'exotic' or 'IJ-type' extensions arise for each pair of integers $\nu_{+}$and $\nu_{-}$, yielding an $N=2\left(\nu_{+}+\nu_{-}\right)$superalgebra of the type discovered recently by Leblanc et al. in non relativistic Chern-Simons theory. For the magnetic monopole the symmetry reduces to $\mathrm{o}(3) \times \operatorname{osp}(1 / 1)$, and for the magnetic vortex it reduces to $\mathrm{o}(2) \times \mathrm{osp}(1 / 2)$.
\end{abstract}

October 1993

CPT-93/P.2912 Journ. Math. Phys. 352516 (1994).

\section{Introduction.}

Recent interest in non-relativistic supersymmetries stems from Chern-Simons theory: as found by Leblanc, Lozano and Min (LLM) [1], non-relativistic Chern-Simons theory in two dimensions admits an $N=2$ conformal supersymmetry, which extends the Schrödinger symmetry discovered previously by Jackiw et al. [2].

$\left(^{1}\right)$ Département de Physique, UFR de Luminy, Université d'Aix-Marseille II and Centre de Physique Théorique CNRS, Case 907, F-13288 MARSEILLE Cedex 09 (France).

$\left({ }^{2}\right)$ Département de Mathématiques, Université de Tours, Parc de Grandmont, F-37200 TOURS (France), 
The story starts in the early seventies, when Niederer, and Hagen, [3] pointed out that the maximal kinematical invariance group of the free, spin-0, Schrödinger equation forms a 13-dimensional Lie group whose Lie algebra is now called the (extended) Schrödinger algebra $\widetilde{\operatorname{sch}}(3)$. This latter contains, in addition to the (centrally extended) Galilei group, two 'conformal' generators, namely dilatations, $\mathcal{D}$, and expansions, $\mathcal{K}$, which close with the Hamiltonian, $\mathcal{H}$, into an $\operatorname{sl}(2, \mathbf{R}) \cong \mathrm{o}(2,1)$ subalgebra [4].

Gauntlett et al. [5] extended this algebra to spin- $\frac{1}{2}$ particles with spin generator $\boldsymbol{\zeta}$. They found five conserved odd generators, namely

$$
\left\{\begin{aligned}
\mathcal{Q} & =\frac{1}{\sqrt{m}} \mathbf{p} \cdot \boldsymbol{\zeta} & & \text { (helicity) } \\
\mathcal{S} & =\sqrt{m}\left(\mathbf{r}-\frac{\mathbf{p}}{m} t\right) \cdot \boldsymbol{\zeta} & & \text { (super-expansion) } \\
\boldsymbol{\Xi} & =\sqrt{m} \boldsymbol{\zeta} & & \text { (spin) }
\end{aligned}\right.
$$

(where $\mathbf{p}=\dot{\mathbf{r}}$ ), which provide an 18-dimensional, $N=1$ supersymmetric extension of the Schrödinger algebra.

Soon after the discovery of the Schrödinger symmetry of the free particle, Niederer [6] found that the harmonic oscillator (described by the bosonic Hamiltonian $H_{B}$ below) also admits this same Schrödinger symmetry - although the generators look quite different, cf. Eq. (7.5). This result has been extended by Beckers et al. [7] to the $n$-dimensional fermionic oscillator with total Hamiltonian

$$
H_{\mathrm{tot}}=H_{B}+H_{F}=\frac{1}{2}\left(\frac{\mathbf{p}^{2}}{m}+m \omega^{2} \mathbf{r}^{2}\right)-i \omega \sum_{a=1}^{n}\left(\zeta_{+}^{a} \zeta_{-}^{a}-\zeta_{-}^{a} \zeta_{+}^{a}\right)
$$

the $\zeta_{ \pm}^{a}(a=1, \ldots, n)$ being the generators of a Clifford algebra. This system has an $N=2$ conformal supersymmetry, with supercharges

$$
\left\{\begin{array}{l}
Q_{ \pm}=(\mathbf{p} \mp i m \omega \mathbf{r}) \cdot \frac{\boldsymbol{\zeta}_{ \pm}}{\sqrt{m}} \\
S_{ \pm}=e^{\mp 2 i \omega t}(\mathbf{p} \pm i m \omega \mathbf{r}) \cdot \frac{\boldsymbol{\zeta}_{ \pm}}{\sqrt{m}} \\
\mathbf{T}_{ \pm}=e^{\mp i \omega t} \sqrt{m} \boldsymbol{\zeta}_{ \pm} .
\end{array}\right.
$$

Note that $H_{B}$ and $H_{F}$ are both bosonic and are separately conserved. For $n=3$, this algebra has 21 generators. 
Non-relativistic Chern-Simons theory in $2+1$ dimensions also has a Schrödinger symmetry [2], extended by Leblanc et al. [1] into a 16-dimensional $N=2$ supersymmetry algebra. But, in two space dimensions, the 'ordinary' $N=2$ superconformal symmetry of the type of Beckers et al. [7] has 18 generators: in particular, there are $N=2$ of each of the ' $\mathcal{Q}$-type', ' $\mathcal{S}$-type' and 'T-type' charges. In the LLM algebra, however, there are $N=2$ ' $\mathcal{Q}$-type' and 'S $\mathcal{S}$-type' supercharges, but just $N / 2=1$ 'T-type' charge. The commutation relations ot the LLM-algebra are similar to, but still different from, those of [7] for $n=2$.

The main result of this paper is to confirm the 'exotic' supersymmetry of Leblanc et al.: we construct, within the symplectic framework [8], extended to the Grassmann case by Berezin, Katz, Kostant, and others [9,10], two types of supersymmetric extensions of the Schrödinger algebra. Our clue is to first imbed the Schrödinger algebra $\widetilde{\operatorname{sch}}(n)$ into $\widetilde{\text { isp }}(n)$, the (central extension of the) algebra of inhomogeneous (or affine) symplectic transformations. But this latter admits a natural supersymmetric extension, namely the affine orthosymplectic algebra $\widetilde{\operatorname{iosp}}(n / m)$ obtained by adding $m$ odd variables. We look therefore for supersymmetric extensions of $\operatorname{sch}(n)$ within $\widetilde{\operatorname{iosp}}(n / m)$. This is reviewed in the first part of the article.

Our I-type extension $\widetilde{\operatorname{sch}}\left(n / N_{+}, N_{-}\right)$exists for any spatial dimension $n$. It is labelled by a pair integers $N_{+}$and $N_{-}$, related to the dimension and the signature of the Grassmann space. Our algebra has $N=N_{+}+N_{-}$of each of the 'Q-type', 'S-type' and 'T-type' charges. If $N_{-}=0\left(N=N_{+}\right)$, we denote this algebra simply by $\widetilde{\operatorname{sch}}(n / N)$.

If, however, space is two-dimensional, we have another 'exotic' or 'IJ-type' extension, we denote by $\widetilde{\operatorname{sch}}_{e}\left(\nu_{+}, \nu_{-}\right)$. It is again labelled by two integers, $\nu_{+}$and $\nu_{-}$, and yields $N=2 \nu=2\left(\nu_{+}+\nu_{-}\right)$of each of the 'Q-type and 'S-type' charges but only $\nu$ 'T-type' charges.

In the second part of our paper, we illustrate our theory on examples.

- Firstly, we recover the $N=1$ super-Schrödinger algebra of Gauntlett et al. [5], in the $I$-type framework.

- Beckers et al.'s oscillator superalgebra [7] corresponds to an I-type extension with $N=N_{+}=2$.

- The superalgebra of Leblanc et al. [1] is obtained as the 'exotic' (' $I J$-type') extension $\widetilde{\operatorname{sch}}_{e}\left(\nu_{+}=1, \nu_{-}=0\right)$.

- Parts of the symmetry algebra may remain unbroken by certain interactions. A celebrated example is the o $(2,1)$ symmetry of the Dirac monopole [11], which extends 
to $\operatorname{osp}(1 / 1)$ when spin is added [12]. Another, more recent, example is provided by the magnetic vortex, which also carries the o $(2,1)$ bosonic symmetry [13]. Now, the Hamiltonian admits two distinct square roots [14] that combine with the bosonic algebra into an osp(1/2) sub-superalgebra of our 'exotic' super-Schrödinger algebra [15] as illustrated by section 9 .

\section{The orthosymplectic algebra.}

Let us start with a supervector space $E=E_{0} \oplus E_{1}$; the endomorphisms of $E$ admit the even/odd decomposition

$$
Z=\left(\begin{array}{cc}
A & B \\
C & D
\end{array}\right)=\left(\begin{array}{cc}
A & 0 \\
0 & D
\end{array}\right)+\left(\begin{array}{cc}
0 & B \\
C & 0
\end{array}\right)
$$

The supercommutator which is defined on homogeneous endomorphisms by $\left[Z, Z^{\prime}\right]=Z Z^{\prime}-$ $(-1)^{|Z| \cdot\left|Z^{\prime}\right|} Z^{\prime} Z$ reads

$$
\begin{aligned}
{\left[\left(\begin{array}{ll}
A & B \\
C & D
\end{array}\right),\right.} & \left.\left(\begin{array}{ll}
A^{\prime} & B^{\prime} \\
C^{\prime} & D^{\prime}
\end{array}\right)\right]= \\
& \left(\begin{array}{ll}
A A^{\prime}-A^{\prime} A+B C^{\prime}+B^{\prime} C & B D^{\prime}-B^{\prime} D+A B^{\prime}-A^{\prime} B \\
C A^{\prime}-C^{\prime} A+D C^{\prime}-D^{\prime} C & D D^{\prime}-D^{\prime} D+C B^{\prime}+C^{\prime} B
\end{array}\right) .
\end{aligned}
$$

From now on we will be dealing with $E=\mathbf{R}^{2 n} \oplus \mathbf{R}_{1}^{m} \equiv \mathbf{R}^{2 n / m}$, globally parametrized by

$$
X=\left(\begin{array}{c}
\mathbf{P} \\
\mathbf{Q}
\end{array}\right) \in \mathbf{R}^{2 n} \quad \text { and } \quad \xi=\left(\begin{array}{c}
\xi^{1} \\
\vdots \\
\xi^{m}
\end{array}\right) \in \mathbf{R}_{1}^{m}
$$

where the $\xi^{\alpha}(\alpha=1, \ldots, m)$ are, along with 1 , the generators of the Grassmann algebra $\Lambda \mathbf{R}^{m}$ and $\mathbf{R}_{1}^{m} \equiv \Lambda^{1} \mathbf{R}^{m}$. Now $\mathbf{R}^{2 n / m}$ carries the canonical symplectic structure of prescribed signature $s$ given by $[9,10]$

$$
\omega=\sum_{j=1}^{n} d P^{j} \wedge d Q^{j}+\frac{1}{2} \sum_{\alpha=1}^{m} \varepsilon_{\alpha} d \xi^{\alpha} \wedge d \xi^{\alpha},
$$

where $\varepsilon_{\alpha}= \pm 1$ and $s=\sum \varepsilon_{\alpha}$. Using the anticommutativity of the odd variables, it is easy to check that

a) $\omega$ is superskewsymmetric, i.e. $\omega\left(V, V^{\prime}\right)+(-1)^{|V| \cdot\left|V^{\prime}\right|} \omega\left(V^{\prime}, V\right)=0$ for all homogeneous elements $V, V^{\prime} \in \mathbf{R}^{2 n / m}$; 
b) $\omega$ is nondegenerate since it is represented by the even invertible endomorphism

$$
\left(\begin{array}{ll}
J & 0 \\
0 & G
\end{array}\right)
$$

according to $\omega\left(V_{0}+V_{1}, V_{0}^{\prime}+V_{1}^{\prime}\right)=V_{0}^{T} J V_{0}^{\prime}+V_{1}^{T} G V_{1}^{\prime}$ where

$$
J=\left(\begin{array}{cc}
0 & I_{n} \\
-I_{n} & 0
\end{array}\right) \quad \text { and } \quad G=\left(\begin{array}{ccc}
\varepsilon_{1} & & \\
& \ddots & \\
& & \varepsilon_{m}
\end{array}\right)
$$

c) $\omega$ is exact:

$$
\omega=\frac{1}{2} d(\overline{\mathbf{P}} d \mathbf{Q}-d \overline{\mathbf{P}} \mathbf{Q}+\bar{\xi} d \xi)
$$

In the last expression, the 'overline' stands for the adjoint with respect to a metric, e.g. $\overline{\mathbf{P}} \equiv \mathbf{P}^{T}$ and $\bar{\xi} \equiv \xi^{T} G$. We will systematically use this notation in the sequel. $\left({ }^{1}\right)$

Let us now recall the definition [9-11] of the orthosymplectic algebra $\operatorname{osp}(n / m)$ which consists of those endomorphisms $Z$ of $\mathbf{R}^{2 n / m}$ whose homogeneous components verify $\omega\left(Z V, V^{\prime}\right)+(-1)^{|Z| \cdot|V|} \omega\left(V, Z V^{\prime}\right)=0$ for all homogeneous elements $V, V^{\prime} \in \mathbf{R}^{2 n / m}$. In other words, an endomorphism $Z$ of the form (2.1) is orthosymplectic for the supersymplectic structure $\omega=\frac{1}{2}(d \bar{X} \wedge J d X+d \bar{\xi} \wedge d \xi)$ of $\mathbf{R}^{2 n / m}$ with signature $s=m_{+}-m_{-}$ if

$$
d(\delta \bar{X}) \wedge J d X+d \bar{X} \wedge J d(\delta X)+d(\delta \bar{\xi}) \wedge d \xi+d \bar{\xi} \wedge d(\delta \xi)=0
$$

where $\delta X=A X+B \xi, \delta \xi=C X+D \xi$, that is if $\bar{A} J+J A=0, C=\bar{B} J$ and $\bar{D}+D=0$. We get therefore the superalgebra

$$
\operatorname{osp}\left(n / m_{+}, m_{-}\right)=\left\{\left(\begin{array}{cc}
A & B \\
\bar{B} J & D
\end{array}\right) \mid A \in \operatorname{sp}(n, \mathbf{R}), B \in L\left(\mathbf{R}_{1}^{m}, \mathbf{R}^{n}\right), D \in \mathrm{o}\left(\mathrm{m}_{+}, \mathrm{m}_{-}\right)\right\}
$$

whose multiplication law is given by (2.2).

(1) If $M \in L\left(\left(\mathbf{R}^{p}, G\right),\left(\mathbf{R}^{q}, H\right)\right)$ then $\bar{M} \equiv G^{-1} M^{T} H$. 


\section{The Schrödinger algebra.}

We first recover the Schrödinger algebra [3,4] in a novel way, namely as a subalgebra of the extended affine symplectic algebra $\widetilde{\operatorname{isp}}(n)$. This is achieved by identifying $\mathbf{R}^{2 n}$ with $\mathbf{R}^{n} \otimes \mathbf{R}^{2}$ (i.e. with the $n \times 2$ matrices) via

$$
\left(\begin{array}{l}
\mathbf{P} \\
\mathbf{Q}
\end{array}\right) \longmapsto(\mathbf{P Q}) \text {. }
$$

One shows now that the linear transformations

$$
(\mathbf{P Q}) \longmapsto A(\mathbf{P Q}) B^{-1}
$$

are symplectic if $A \in \mathrm{o}(\mathrm{n})$ and $B \in \mathrm{SL}(2, \mathbf{R})$. The corresponding Lie algebra is isomorphic to

$$
\left\{\left(\begin{array}{cc}
A+a I_{n} & b I_{n} \\
c I_{n} & A-a I_{n}
\end{array}\right) \mid A \in \mathrm{o}(\mathrm{n}) ; \mathrm{a}, \mathrm{b}, \mathrm{c} \in \mathbf{R}\right\} \cong \mathrm{o}(\mathrm{n}) \times \operatorname{sl}(2, \mathbf{R}) \subset \operatorname{sp}(\mathrm{n}, \mathbf{R}) .
$$

Note that the 'dual pair' $\mathrm{o}(\mathrm{n}) \times \mathrm{sl}(2, \mathbf{R})$ is the homogeneous Schrödinger algebra [3,4]. The full centrally extended Schrödinger Lie algebra [3,4] is conveniently obtained $[8,12]$ by considering rather the canonical contact structure on $\mathbf{R}^{2 n+1}$,

$$
\varpi=\frac{1}{2}(\overline{\mathbf{P}} d \mathbf{Q}-d \overline{\mathbf{P}} \mathbf{Q})+d s,
$$

and look for the infinitesimal affine contact transformations

$$
\left(\begin{array}{c}
\mathbf{P} \\
\mathbf{Q} \\
s
\end{array}\right) \longmapsto \delta\left(\begin{array}{c}
\mathbf{P} \\
\mathbf{Q} \\
s
\end{array}\right)
$$

which Lie-transport the 1 -form $\varpi=0$,

$$
\left.\frac{1}{2}(\delta \overline{\mathbf{P}}) d \mathbf{Q}+\overline{\mathbf{P}} d(\delta \mathbf{Q})-d \overline{\mathbf{P}}(\delta \mathbf{Q})-d(\delta \overline{\mathbf{P}}) \mathbf{Q}\right)+d(\delta s)=0,
$$

and extend $\mathrm{o}(\mathrm{n}) \times \operatorname{sl}(2, \mathbf{R})$. One readily finds

$$
\delta\left(\begin{array}{c}
\mathbf{P} \\
\mathbf{Q} \\
s \\
1
\end{array}\right)=\left(\begin{array}{cccc}
A+a I_{n} & b I_{n} & 0 & \mathbf{V} \\
c I_{n} & A-a I_{n} & 0 & \mathbf{W} \\
\frac{1}{2} \overline{\mathbf{W}} & -\frac{1}{2} \overline{\mathbf{V}} & 0 & u \\
0 & 0 & 0 & 0
\end{array}\right)\left(\begin{array}{c}
\mathbf{P} \\
\mathbf{Q} \\
s \\
1
\end{array}\right)
$$

where $A \in \mathrm{o}(\mathrm{n}) ; \mathrm{a}, \mathrm{b}, \mathrm{c}, \mathrm{u} \in \mathbf{R} ; \mathbf{V}, \mathbf{W} \in \mathbf{R}^{\mathrm{n}}$ which defines the extended Schrödinger algebra $\widetilde{\operatorname{sch}}(n)$, the central $\mathbf{R}$-extension of

$$
\operatorname{sch}(n) \cong(\mathrm{o}(\mathrm{n}) \times \operatorname{sl}(2, \mathbf{R})) \mathrm{S} \mathbf{R}^{2 n}
$$




\section{Schrödinger superalgebras: I-type extensions.}

Let us now merge the two previous structures into a single one, viz. the canonical contact structure on $\mathbf{R}^{2 n+1 / m}[10]$ given by the even 1 -form

$$
\alpha=\frac{1}{2}(\overline{\mathbf{P}} d \mathbf{Q}-d \overline{\mathbf{P}} \mathbf{Q})+\frac{1}{2} \bar{\xi} d \xi+d s
$$

such that $\omega=d \alpha$. A tedious but routine calculation shows that the infinitesimal affine contact transformations are given by

$$
\delta\left(\begin{array}{c}
\mathbf{P} \\
\mathbf{Q} \\
s \\
1 \\
\xi
\end{array}\right)=\left(\begin{array}{ccccc}
A & B & 0 & \mathbf{V} & -\Sigma \\
C & -\bar{A} & 0 & \mathbf{W} & -\Theta \\
\frac{1}{2} \overline{\mathbf{W}} & -\frac{1}{2} \overline{\mathbf{V}} & 0 & u & -\frac{1}{2} \bar{\Psi} \\
0 & 0 & 0 & 0 & 0 \\
\bar{\Theta} & -\bar{\Sigma} & 0 & \Psi & D
\end{array}\right)\left(\begin{array}{c}
\mathbf{P} \\
\mathbf{Q} \\
s \\
1 \\
\xi
\end{array}\right)
$$

with $B=\bar{B}, C=\bar{C}, u \in \mathbf{R} ; \mathbf{V}, \mathbf{W} \in \mathbf{R}^{n} ; \Sigma, \Theta \in L\left(\mathbf{R}_{1}^{m}, \mathbf{R}^{n}\right), \Psi \in \mathbf{R}_{1}^{m}$ and $\bar{D}+D=0$, i.e. $D \in \mathrm{o}\left(\mathrm{m}_{+}, \mathrm{m}_{-}\right)$. This is, indeed, the extended affine orthosymplectic superalgebra $\widetilde{\operatorname{iosp}}\left(n / m_{+}, m_{-}\right)$, spanned by the vector spaces $\widetilde{\operatorname{isp}}(n, \mathbf{R}), \mathrm{o}\left(\mathrm{m}_{+}, \mathrm{m}_{-}\right)$, and $L\left(\mathbf{R}_{1}^{m}, \mathbf{R}^{2 n+1}\right)$.

Let us now look for Lie superalgebras that extend nontrivially the Schrödinger algebra $\widetilde{\operatorname{sch}}(n)$ in $(3.4)$ within $\widetilde{\operatorname{iosp}}\left(n / m_{+}, m_{-}\right)$. Thus, the commutator of two infinitesimal transformations (4.2) should close according to (3.4); the crucial condition is that $A, B$ and $C$ in Eq. (4.2) be of the same form as in Eq. (3.4). By Eq. (2.2), this requires

$$
\left\{\begin{array}{l}
\Sigma \overline{\Theta^{\prime}}+\Sigma^{\prime} \bar{\Theta}+\Theta \overline{\Sigma^{\prime}}+\Theta^{\prime} \bar{\Sigma}=a^{\prime \prime} I_{n} \\
\Sigma \overline{\Sigma^{\prime}}+\Sigma^{\prime} \bar{\Sigma}=b^{\prime \prime} I_{n} \\
\Theta \overline{\Theta^{\prime}}+\Theta^{\prime} \bar{\Theta}=c^{\prime \prime} I_{n}
\end{array}\right.
$$

for some scalars $a^{\prime \prime}, b^{\prime \prime}$ and $c^{\prime \prime}$. One way of satisfying these conditions is to assume that the matrices split into $N$ blocks,

$$
G=\left(\begin{array}{ccc}
\epsilon_{1} I_{n} & & \\
& \ddots & \\
& & \epsilon_{N} I_{n}
\end{array}\right),
$$

so that $m=n N$ and $\xi=\left(\begin{array}{c}\boldsymbol{\xi}_{1} \\ \vdots \\ \boldsymbol{\xi}_{N}\end{array}\right)$ with $\boldsymbol{\xi}_{j} \in \mathbf{R}_{1}^{n}$, supplemented by the ' $I$-type' Ansatz

$$
\Sigma=\left(\sigma^{1} I_{n} \ldots \sigma^{N} I_{n}\right) \quad \text { and } \quad \Theta=\left(\theta^{1} I_{n} \ldots \theta^{N} I_{n}\right),
$$

where $\sigma^{j}, \theta^{k} \in \mathbf{R}_{1}$ for all $j, k=1, \ldots, N$. 
Let $N_{+}$(resp. $N_{-}$) be the number of blocks in (4.4) with positive (resp. negative) sign and $N=N_{+}+N_{-}$. For each pair $\left(N_{+}, N_{-}\right)$, we get a supersymmetric extension of the Schrödinger algebra we call $\left(N_{+}, N_{-}\right)$-Schrödinger superalgebra of I-type, and denote by $\widetilde{\operatorname{sch}}\left(n / N_{+}, N_{-}\right)$. It consists of endomorphisms in $\operatorname{sl}(2 n+2 / n N)$ of the form

$$
Z=\left(\begin{array}{cccccccc}
A+a I_{n} & b I_{n} & 0 & \mathbf{V} & -\sigma^{1} I_{n} & . & \ldots & -\sigma^{N} I_{n} \\
c I_{n} & A-a I_{n} & 0 & \mathbf{W} & -\theta^{1} I_{n} & . & \ldots & -\theta^{N} I_{n} \\
\frac{1}{2} \overline{\mathbf{W}} & -\frac{1}{2} \overline{\mathbf{V}} & 0 & u & -\frac{1}{2} \overline{\mathbf{\Psi}}^{1} & . & \ldots & -\frac{1}{2} \overline{\mathbf{\Psi}}^{N} \\
0 & 0 & 0 & 0 & 0 & . & \ldots & 0 \\
\theta_{1} I_{n} & -\sigma_{1} I_{n} & 0 & \boldsymbol{\Psi}_{1} & A & R_{1}^{2} I_{n} & \ldots & R_{1}^{N} I_{n} \\
\cdot & . & . & . & R_{2}^{1} I_{n} & A & \ldots & . \\
\vdots & \vdots & \vdots & \vdots & \vdots & \vdots & \ddots & \vdots \\
\theta_{N} I_{n} & -\sigma_{N} I_{n} & 0 & \boldsymbol{\Psi}_{N} & R_{N}^{1} I_{n} & . & \ldots & A
\end{array}\right)
$$

with $A \in \mathrm{o}(\mathrm{n}) ; a, b, c, u \in \mathbf{R} ; \mathbf{V}, \mathbf{W} \in \mathbf{R}^{n} ; R=\left(R_{j}^{k}\right) \in \mathrm{o}\left(\mathrm{N}_{+}, \mathrm{N}_{-}\right) ; \sigma_{j} \equiv \epsilon_{j} \sigma^{j}, \theta_{j} \equiv \epsilon_{j} \theta^{j} \in$ $\mathbf{R}_{1}, \boldsymbol{\Psi}_{j} \in \mathbf{R}_{1}^{n}, \overline{\boldsymbol{\Psi}}^{j} \equiv \epsilon_{j} \boldsymbol{\Psi}_{j}^{T}($ all $j, k=1, \ldots, N)$. The supercommutators $Z^{\prime \prime}=\left[Z, Z^{\prime}\right] \mathrm{read}$

$$
\left\{\begin{aligned}
A^{\prime \prime} & =A A^{\prime}-A^{\prime} A \\
a^{\prime \prime} & =b c^{\prime}-b^{\prime} c-\sum_{j=1}^{N}\left(\theta_{j} \sigma^{\prime j}+\theta_{j}^{\prime} \sigma^{j}\right) \\
b^{\prime \prime} & =2\left(a b^{\prime}-a^{\prime} b\right)+2 \sum_{j=1}^{N} \sigma_{j} \sigma^{j} \\
c^{\prime \prime} & =2\left(c a^{\prime}-c^{\prime} a\right)-2 \sum_{j=1}^{N} \theta_{j} \theta^{j} \\
\mathbf{V}^{\prime \prime} & =A \mathbf{V}^{\prime}-A^{\prime} \mathbf{V}+a \mathbf{V}^{\prime}-a^{\prime} \mathbf{V}+b \mathbf{W}^{\prime}-b^{\prime} \mathbf{W}-\sum_{j=1}^{N}\left(\mathbf{\Psi}_{j} \sigma^{\prime j}+\mathbf{\Psi}_{j}^{\prime} \sigma^{j}\right) \\
\mathbf{W}^{\prime \prime} & =A \mathbf{W}^{\prime}-A^{\prime} \mathbf{W}-a \mathbf{W}^{\prime}+a^{\prime} \mathbf{W}+c \mathbf{V}^{\prime}-c^{\prime} \mathbf{V}-\sum_{j=1}^{N}\left(\mathbf{\Psi}_{j} \theta^{\prime j}+\mathbf{\Psi}_{j}^{\prime} \theta^{j}\right) \\
u^{\prime \prime} & =-\overline{\mathbf{V}} \mathbf{W}^{\prime}+\overline{\mathbf{V}^{\prime}} \mathbf{W}_{j}-\sum_{j=1}^{N} \overline{\mathbf{\Psi}}^{j} \mathbf{\Psi}_{j}^{\prime} \\
R^{\prime \prime}{ }_{j}^{k} & =-\sum_{i=1}^{N}\left(R_{i}^{k} R_{j}^{\prime i}-R_{i}^{\prime k} R_{j}^{i}\right)+\theta^{k} \sigma_{j}^{\prime}-\theta^{\prime k} \sigma_{j}-\sigma^{k} \theta_{j}^{\prime}-\sigma^{\prime k} \theta_{j}
\end{aligned}\right.
$$


for the bosonic part, and

$$
\left\{\begin{array}{l}
\sigma^{\prime \prime j}=a \sigma^{j}-a^{\prime} \sigma^{j}+b \theta^{\prime j}-b^{\prime} \theta^{j}-\sum_{k=1}^{N}\left(R_{k}^{j} \sigma^{\prime k}-R_{k}^{\prime j} \sigma^{k}\right), \\
\theta^{\prime \prime j}=-a \theta^{\prime j}+a^{\prime} \theta^{j}+c \sigma^{\prime j}-c^{\prime} \sigma^{j}-\sum_{k=1}^{N}\left(R_{k}^{j} \theta^{\prime k}-R_{k}^{\prime j} \theta^{k}\right), \\
\mathbf{\Psi}_{j}^{\prime \prime}=A \boldsymbol{\Psi}_{j}^{\prime}-A^{\prime} \mathbf{\Psi}_{j}-\sigma_{j} \mathbf{W}^{\prime}+\sigma_{j}^{\prime} \mathbf{W}+\theta_{j} \mathbf{V}^{\prime}-\theta_{j}^{\prime} \mathbf{V}-\sum_{k=1}^{N}\left(\mathbf{\Psi}_{k} R_{j}^{\prime k}-\boldsymbol{\Psi}_{k}^{\prime} R_{j}^{k}\right) .
\end{array}\right.
$$

for the fermionic part.

The components of the associated supermoment map [10],

$$
\mu: \mathbf{R}^{2 n+1 / n N} \longrightarrow \widetilde{\operatorname{sch}}\left(n / N_{+}, N_{-}\right)^{*}
$$

are found using the expression

$$
\langle\mu, Z\rangle=\imath\left(Z_{E}\right) \alpha
$$

the infinitesimal action $Z_{E}$ of $Z$ (given by (4.6)) on $E=\mathbf{R}^{2 n+1 / n N}$ being defined in (4.2). Putting

$$
\begin{aligned}
\langle\mu, Z\rangle= & \frac{1}{2} \operatorname{Tr}(\mathcal{J} A)+\mathcal{H} c-\mathcal{D} a-\mathcal{K} b-\overline{\mathcal{G}} \mathbf{V}+\overline{\mathcal{P}} \mathbf{W}+\mathcal{M} u-\frac{1}{2} \mathcal{H}_{j k} R^{j k} \\
& +\mathcal{Q}_{j} \theta^{j}-\mathcal{S}_{j} \sigma^{j}+\overline{\boldsymbol{\Xi}}^{j} \boldsymbol{\Psi}_{j}
\end{aligned}
$$

where we have adopted the Einstein summation convention and used the metric

$$
g_{j k}=\epsilon_{j} \delta_{j k}
$$

to raise and lower fermionic indices, we claim that

$$
\left\{\begin{array}{lll}
\mathcal{J} & =\mathbf{Q} \overline{\mathbf{P}}-\mathbf{P} \overline{\mathbf{Q}}-\boldsymbol{\xi}_{j} \overline{\boldsymbol{\xi}}^{j}, & \mathcal{Q}_{j}=\overline{\mathbf{P}} \boldsymbol{\xi}_{j} \\
\mathcal{H} & =\frac{1}{2}\|\mathbf{P}\|^{2}, & \mathcal{S}_{j}=\overline{\mathbf{Q}} \boldsymbol{\xi}_{j} \\
\mathcal{D} & =\overline{\mathbf{P}} \mathbf{Q}, & \boldsymbol{\Xi}_{j}=\boldsymbol{\xi}_{j} \\
\mathcal{K} & =\frac{1}{2}\|\mathbf{Q}\|^{2}, & \\
\mathcal{G} & =\mathbf{Q} \\
\mathcal{P} & =\mathbf{P} \\
\mathcal{M} & =1, \\
\mathcal{H}_{k}^{j} & =\overline{\boldsymbol{\xi}}^{j} \boldsymbol{\xi}_{k},
\end{array}\right.
$$

where $j, k=1, \ldots, N$. The components of $\mu$ plainly generate, under super-Poisson brackets we still denote by $[\cdot, \cdot]$, a superalgebra isomorphic to $\widetilde{\operatorname{sch}}\left(n / N_{+}, N_{-}\right)$. 
The crucial formula $\left({ }^{2}\right)$

$$
\left[\langle\mu, Z\rangle,\left\langle\mu, Z^{\prime}\right\rangle\right]=-\left\langle\mu,\left[Z, Z^{\prime}\right]\right\rangle
$$

yields, using the commutation relations $(4.7,8)$, the 'even' Poisson brackets

$$
\left[\mathcal{J}_{a b}, \mathcal{J}_{c d}\right]=\delta_{c b} \mathcal{J}_{a d}-\delta_{c a} \mathcal{J}_{b d}+\delta_{d b} \mathcal{J}_{c a}-\delta_{d a} \mathcal{J}_{c b}
$$

and

$$
\begin{aligned}
& {[\mathcal{H}, \mathcal{D}]=2 \mathcal{H} \quad[\mathcal{H}, \mathcal{K}]=\mathcal{D} \quad[\mathcal{D}, \mathcal{K}]=2 \mathcal{K}} \\
& {\left[\mathcal{J}_{a b}, \mathcal{H}\right]=0 \quad\left[\mathcal{J}_{a b}, \mathcal{D}\right]=0 \quad\left[\mathcal{J}_{a b}, \mathcal{K}\right]=0} \\
& {\left[\mathcal{G}_{a}, \mathcal{G}_{b}\right]=0 \quad\left[\mathcal{P}_{a}, \mathcal{P}_{b}\right]=0 \quad\left[\mathcal{P}_{a}, \mathcal{G}^{b}\right]=\delta_{a}^{b} \mathcal{M}} \\
& {\left[\mathcal{G}_{a}, \mathcal{J}^{b c}\right]=\delta_{a}^{b} \mathcal{G}^{c}-\delta_{a}^{c} \mathcal{G}^{b} \quad\left[\mathcal{P}_{a}, \mathcal{J}^{b c}\right]=\delta_{a}^{b} \mathcal{P}^{c}-\delta_{a}^{c} \mathcal{P}^{b}} \\
& {\left[\mathcal{H}, \mathcal{G}_{a}\right]=\mathcal{P}_{a} \quad\left[\mathcal{H}, \mathcal{P}_{a}\right]=0 \quad\left[\mathcal{D}, \mathcal{G}_{a}\right]=\mathcal{G}_{a}} \\
& {\left[\mathcal{D}, \mathcal{P}_{a}\right]=-\mathcal{P}_{a} \quad\left[\mathcal{K}, \mathcal{G}_{a}\right]=0 \quad\left[\mathcal{K}, \mathcal{P}_{a}\right]=-\mathcal{G}_{a} \text {, }}
\end{aligned}
$$

where $a, b, c, d=1, \ldots, n,(\mathcal{M}$ commutes with everything $)$. These are the commutation relations of $(\mathrm{o}(\mathrm{n}) \times \mathrm{o}(2,1)) \mathrm{S} \mathrm{h}(\mathrm{n})$, i.e. that of the extended Schrödinger algebra $\widetilde{\operatorname{sch}}(n)$. The $\mathcal{H}_{i j}$ 's commute with all other bosonic generators and, themselves, generate o $\left(\mathrm{N}_{+}, \mathrm{N}_{-}\right)$:

$$
\left[\mathcal{H}_{i j}, \mathcal{H}_{k \ell}\right]=-g_{k j} \mathcal{H}_{i \ell}+g_{k i} \mathcal{H}_{j \ell}-g_{\ell j} \mathcal{H}_{k i}+g_{\ell i} \mathcal{H}_{k j}
$$

where $i, j, k, \ell=1, \ldots, N$. The bosonic commutation relations are hence those of the direct product of the extended Schrödinger algebra with $\mathrm{o}\left(\mathrm{N}_{+}, \mathrm{N}_{-}\right)$. They are supplemented with the relations involving 'fermionic' (i.e. odd) component. Firstly, we have

$$
\begin{array}{llll}
{\left[\mathcal{Q}_{j}, \mathcal{D}\right]} & =\mathcal{Q}_{j} & & {\left[\mathcal{Q}_{j}, \mathcal{K}\right]=\mathcal{S}_{j}} \\
{\left[\mathcal{Q}_{j}, \mathcal{H}\right]} & =0 & {\left[\mathcal{Q}_{i}, \mathcal{H}\right.} & \left.=g_{i k}\right] \\
\left.\mathcal{S}_{j}, \mathcal{D}\right] & =-\mathcal{S}_{j} \mathcal{Q}_{k} \\
{\left[\mathcal{S}_{j}, \mathcal{H}\right]} & =-\mathcal{Q}_{j} & {\left[\mathcal{S}_{j}, \mathcal{K}\right]=0} \\
{\left[\mathcal{Q}_{j}, \mathcal{Q}_{k}\right]=-2 g_{j k} \mathcal{H}} & {\left[\mathcal{S}_{i}, \mathcal{H}_{j k}\right]=g_{i k} S_{j}-g_{i j} \mathcal{S}_{k}} \\
{\left[\mathcal{Q}_{j}, \mathcal{S}_{k}\right]=-g_{j k} \mathcal{D}+\mathcal{H}_{j k},} & {\left[\mathcal{S}_{j}, \mathcal{S}_{k}\right]=-2 g_{j k} \mathcal{K}}
\end{array}
$$

which says that the $\mathcal{H}, \mathcal{D}, \mathcal{K}, \mathcal{H}_{j k}$ close with $\mathcal{Q}_{j}, \mathcal{S}_{j}$ into $\operatorname{osp}\left(1 / N_{+}, N_{-}\right)$.

$\left({ }^{2}\right)$ According to our convention, $\left[Z_{E}, Z_{E}^{\prime}\right] \equiv-\left[Z, Z^{\prime}\right]_{E}$. 
Next, according to

$$
\left[\mathcal{Q}_{j}, \mathcal{J}_{a b}\right]=0, \quad\left[\mathcal{S}_{j}, \mathcal{J}_{a b}\right]=0,
$$

the 'homogeneous super-Schrödinger algebra' is the direct product o $(n) \times \operatorname{osp}\left(1 / \mathrm{N}_{+}, \mathrm{N}_{-}\right)$. The commutators of translations and super-translations,

$$
\left[\mathcal{P}_{a}, \Xi_{j}^{b}\right]=0, \quad\left[\mathcal{G}_{a}, \Xi_{j}^{b}\right]=0, \quad\left[\Xi_{j}^{a}, \Xi_{k}^{b}\right]=-g_{j k} \delta^{a b} \mathcal{M},
$$

define the super-Heisenberg algebra $\mathrm{h}\left(n / N_{+}, N_{-}\right)$Beckers et al. denote by $\operatorname{sh}(n)$. Finally,

$$
\begin{array}{llll}
{\left[\Xi_{j}^{a}, \mathcal{J}^{b c}\right]} & =\delta^{a b} \Xi_{j}^{c}-\delta^{a c} \Xi_{j}^{b} & & \\
{\left[\mathcal{H}, \Xi_{j}^{a}\right]} & =0 & & {\left[\mathcal{D}, \Xi_{j}^{a}\right]=0} \\
{\left[\mathcal{K}, \Xi_{j}^{a}\right]} & =0 & & {\left[\mathcal{H}_{j k}, \Xi_{i}^{a}\right]=g_{i j} \Xi_{k}^{a}-g_{i k} \Xi_{j}^{a}} \\
{\left[\mathcal{Q}_{j}, \mathcal{G}^{a}\right]} & =\Xi_{j}^{a} & & {\left[\mathcal{Q}_{j}, \mathcal{P}\right]=0} \\
{\left[\mathcal{S}_{j}, \mathcal{G}_{a}\right]} & =0 & {\left[\mathcal{S}_{j}, \mathcal{P}^{a}\right]} & =-\Xi_{j}^{a} \\
{\left[\mathcal{Q}_{j}, \Xi_{k}^{a}\right]} & =-g_{j k} \mathcal{P}^{a} & {\left[S_{j}, \Xi_{k}^{a}\right]} & =-g_{j k} \mathcal{G}^{a}
\end{array}
$$

fixes the relation between the homogeneous and inhomogeneous parts as a semidirect product. In conclusion, our superalgebra has the following structure:

$$
\widetilde{\operatorname{sch}}\left(n / N_{+}, N_{-}\right)=\left(\mathrm{o}(\mathrm{n}) \times \operatorname{osp}\left(1 / \mathrm{N}_{+}, \mathrm{N}_{-}\right)\right) \circledast \mathrm{h}\left(n / N_{+}, N_{-}\right),
$$

generalizing what Beckers et al. [7] found for $N=N_{+}=2$. In most frequent cases, when all epsilon's are equal to 1 and $N=N_{+}$, we denote our Schrödinger algebras simply by $\operatorname{sch}(n / N)$.

\section{Discussion.}

Let us recall [4] that the $\mathrm{SL}(2, \mathbf{R})$ subgroup of the Schrödinger group actually shows up in the presymplectic framework over space-time as a covering group of the projective transformations of the time axis. Our $I$-type Ansatz, (4.4-5) amounts to decomposing $\mathbf{R}^{2 n}$ by viewing it as a tensor product,

$$
\mathbf{R}^{2 n} \cong \mathbf{R}^{n} \otimes \mathbf{R}^{2}
$$

and super-extending it by extending the 'time-like' factor $\mathbf{R}^{2}$ into $\mathbf{R}^{2 / N}$, so that the supersymplectic phase space becomes

$$
\mathbf{R}^{2 n / n N} \cong \mathbf{R}^{n} \otimes \mathbf{R}^{2 / N} .
$$


The structure of the (unextended) $I$-type superalgebra $\operatorname{sch}\left(n / N_{+}, N_{-}\right)$becomes clear by writing its action on the super-symplectic space in the form

$$
\delta\left(\mathbf{P Q} \boldsymbol{\xi}_{1} \ldots \boldsymbol{\xi}_{N}\right)=A\left(\mathbf{P Q} \boldsymbol{\xi}_{1} \ldots \boldsymbol{\xi}_{N}\right)+\left(\mathbf{P Q} \boldsymbol{\xi}_{1} \ldots \boldsymbol{\xi}_{N}\right) B+C
$$

where (cf. (4.6) for the notation)

$$
\left\{\begin{array}{l}
A \in \mathrm{o}(\mathrm{n}) \\
B=\left(\begin{array}{ccc}
a & c & \bar{\theta} \\
b & -a & -\bar{\sigma} \\
\sigma & \theta & R
\end{array}\right) \in \operatorname{osp}\left(1 / N_{+}, N_{-}\right) \\
C=\left(\mathbf{V W} \boldsymbol{\Psi}_{1} \ldots \boldsymbol{\Psi}_{N}\right) \in \mathbf{R}^{2 n / n N} .
\end{array}\right.
$$

We note that the $I$-type extension amounts hence to super-extending the conformal subalgebra $\operatorname{sl}(2, \mathbf{R}) \cong \operatorname{osp}(1 / 0)$ into $\operatorname{osp}\left(1 / N_{+}, N_{-}\right)$. In other words, it consists of those automorphisms that respect the 'space-supertime' factorization (4.21) much in the same way as the Schrödinger algebra $\operatorname{sch}(n)$ does for the 'space-time' factorization (4.20).

\section{Schrödinger superalgebras: exotic ( $I J$-type) extensions.}

Now we try to find other supersymmetric extensions of the Schrödinger algebra. Let us hence return to the conditions (4.3), and inquire under what circumstances the second one, for example,

$$
\Sigma \bar{\Sigma}^{\prime}+\Sigma^{\prime} \bar{\Sigma}=\lambda I_{n}
$$

$(\lambda \in \mathbf{R})$ can be satisfied by $\Sigma$ 's of the form

$$
\Sigma=\left(\Sigma^{1} \ldots \Sigma^{\nu}\right)
$$

where $\Sigma^{j}$ is in $L\left(\mathbf{R}^{n}, \mathbf{R}^{n}\right)$ for $j=1, \ldots, \nu$. Such a matrix $\Sigma^{j}$ can be decomposed into its symmetric and antisymmetric parts (with repect to the metric $G_{j}=\epsilon_{j} I_{n}$ ), and condition (5.1), applied to $\Sigma$ and $\Sigma^{\prime}$ with $\Sigma^{\prime k}=\delta^{j k} I_{n}$, entails that any $\Sigma^{j}$ is necessarily of the form $\sigma^{j} I_{n}+\Omega^{j}$, where $\Omega^{j}+\overline{\Omega^{j}}=0$. Thus $\Omega^{j}$ is an orthogonal matrix. Now Eq. (5.1) requires

$$
\left(\Omega^{j}\right)^{2}=\lambda^{j} I_{n}
$$


Thus, in the generic case $\lambda^{j} \neq 0, \Omega^{j}$ is invertible, so that space has to be even dimensional. Let $\left\{\Omega_{K}\right\}$ be a basis of the subspace spanned by those orthogonal matrices which satisfy Eq. (5.3). Then it is easy to verify that all three relations in Eq. (4.3) hold if

$$
\left\{\begin{array} { l } 
{ \Sigma = ( \Sigma ^ { 1 } \ldots \Sigma ^ { \nu } ) } \\
{ \Theta = ( \Theta ^ { 1 } \ldots \Theta ^ { \nu } ) , }
\end{array} \quad \text { where } \quad \left\{\begin{array}{l}
\Sigma^{j}=\sigma^{j} I_{n}+\sigma_{*}^{j K} \Omega_{K} \\
\Theta^{j}=\theta^{j} I_{n}+\theta_{*}^{j K} \Omega_{K} .
\end{array}\right.\right.
$$

Then some algebra yields the crucial condition

$$
\sigma_{*}^{j K}\left(\Omega_{K} A-A \Omega_{K}\right)=0
$$

for all $A \in \mathrm{o}(n)$. Thus, if $n>2$, the only possibility that wouldn't break the o $(n)$ symmetry, is $\sigma_{*}^{j K}=0$. In other words, for $n>2$, the $I$-type extension considered in Section 4 is the only possibility consistent with the Ansatz (5.2).

If, however the space is two-dimensional, the rotation group is Abelian, and one can have a non-trivial $\Omega$, namely

$$
\Omega=\sigma_{*} J
$$

$J$ being the generator of rotations in the plane, $J=\left(\begin{array}{cc}0 & 1 \\ -1 & 0\end{array}\right)$.

Let us hence examine the planar case $n=2$ in some more detail. Now the Grassmann space is $m=2 \nu$-dimensional. Then the matrix $D$ in (4.2) can be decomposed into $2 \times 2$ blocks,

$$
D=\left(\begin{array}{cccc}
D_{1}^{1} & D_{1}^{2} & \ldots & \cdot \\
D_{2}^{1} & \cdot & & \cdot \\
\vdots & & \ddots & \vdots \\
. & . & \ldots & D_{\nu}^{\nu}
\end{array}\right)
$$

Since $D$ is orthogonal, $D \in \mathrm{o}\left(2 \nu_{+}, 2 \nu_{-}\right)$, each $2 \times 2$ block is further seen to be of the form $D_{k}^{j}=R_{k}^{j} I+S_{k}^{j} J$ where $I=I_{2},\left(R_{k}^{j}\right)$ is in $o(\nu)$ (a shorthand for o $\left(\nu_{+}, \nu_{-}\right)$), and $\left(S_{k}^{j}\right)$ is a symmetric $\nu \times \nu$ matrix. Hence we have proved that, for $n=2$, the Ansatz (4.5) can be generalized $($ see $(5.4,6))$ to

$$
\left\{\begin{array}{l}
\Sigma=\left(\sigma^{1} I+\sigma_{*}^{1} J \ldots \sigma^{\nu} I+\sigma_{*}^{\nu} J\right) \\
\Theta=\left(\theta^{1} I+\theta_{*}^{1} J \ldots \theta^{\nu} I+\theta_{*}^{\nu} J\right),
\end{array}\right.
$$


yielding the 'exotic' super-Schrödinger algebra which consists of those endomorphisms of $\mathbf{R}^{6 / 2 \nu}$ of the form

$$
Z=\left(\begin{array}{ccccccc}
A+a I & b I & 0 & \mathbf{V} & -\Sigma^{1} & \ldots & -\Sigma^{\nu} \\
c I & A-a I & 0 & \mathbf{W} & -\Theta^{1} & \ldots & -\Theta^{\nu} \\
\frac{1}{2} \overline{\mathbf{W}} & -\frac{1}{2} \overline{\mathbf{V}} & 0 & u & -\frac{1}{2} \overline{\boldsymbol{\Psi}}^{1} & \ldots & -\frac{1}{2} \overline{\boldsymbol{\Psi}}^{\nu} \\
0 & 0 & 0 & 0 & 0 & \ldots & 0 \\
\bar{\Theta}_{1} & -\bar{\Sigma}_{1} & 0 & \boldsymbol{\Psi}_{1} & D_{1}^{1} & \ldots & D_{1}^{\nu} \\
\vdots & \vdots & \vdots & \vdots & \vdots & \ddots & \vdots \\
\bar{\Theta}_{\nu} & -\bar{\Sigma}_{\nu} & 0 & \boldsymbol{\Psi}_{\nu} & D_{\nu}^{1} & \ldots & D_{\nu}^{\nu}
\end{array}\right)
$$

where $A=\omega J ; a, b, c, u \in \mathbf{R} ; \mathbf{V}, \mathbf{W} \in \mathbf{R}^{2} ; \Sigma^{j}=\sigma^{j} I+\sigma_{*}^{j} J, \Theta^{j}=\theta^{j} I+\theta_{*}^{j} J ; \mathbf{\Psi}_{j} \in \mathbf{R}_{1}^{2}$; the $2 \times 2$ block matrices $D_{k}^{j}$ being given for $j, k=1, \ldots, \nu$ by

$$
D_{k}^{j}=R_{k}^{j} I+S_{k}^{j} J
$$

with $R+\bar{R}=0$, i.e. $R \in \mathrm{o}(\nu)$ and $S=\bar{S}$.

The components of the supermoment map are, this time

$$
\begin{aligned}
\langle\mu, Z\rangle= & -\mathcal{J} \omega+\mathcal{H} c-\mathcal{D} a-\mathcal{K} b-\overline{\mathcal{G}} \mathbf{V}+\overline{\mathcal{P}} \mathbf{W}+\mathcal{M} u \\
& -\frac{1}{2} \mathcal{H}_{j k} R^{j k}+\frac{1}{2} \mathcal{L}_{j k} S^{j k}+\mathcal{Q}_{j} \theta^{j}+\mathcal{Q}_{j}^{*} \theta_{*}^{j}-\mathcal{S}_{j} \sigma^{j}-\mathcal{S}_{j}^{*} \sigma_{*}^{j}+\overline{\boldsymbol{\Xi}}^{j} \boldsymbol{\Psi}_{j}
\end{aligned}
$$

with $j, k=1, \ldots, \nu$. (Again, indices in (5.10) are raised and lowered by means of the metric $g_{i j}=\epsilon_{i} \delta_{i j}$.) Explicitly, we get the remarkably symmetric formulæ

$$
\left\{\begin{array}{rlrl}
\mathcal{J} & =\mathbf{Q} \times \mathbf{P}, & \mathcal{Q}_{j}=\mathbf{P} \cdot \boldsymbol{\xi}_{j}, \\
\mathcal{H} & =\frac{1}{2}\|\mathbf{P}\|^{2}, & \mathcal{Q}_{j}^{*}=\mathbf{P} \times \boldsymbol{\xi}_{j}, \\
\mathcal{D} & =\mathbf{P} \cdot \mathbf{Q}, & \mathcal{S}_{j}=\mathbf{Q} \cdot \boldsymbol{\xi}_{j}, \\
\mathcal{K} & =\frac{1}{2}\|\mathbf{Q}\|^{2}, & \mathcal{S}_{j}^{*}=\mathbf{Q} \times \boldsymbol{\xi}_{j}, \\
\mathcal{G} & =\mathbf{Q}, & \boldsymbol{\Xi}_{j}=\boldsymbol{\xi}_{j}, \\
\mathcal{P} & =\mathbf{P}, & & \\
\mathcal{M} & =1, & & \\
\mathcal{H}_{j k} & =\mathcal{H}_{[j k]}=\boldsymbol{\xi}_{j} \cdot \boldsymbol{\xi}_{k}, & \\
\mathcal{L}_{j k}=\mathcal{L}_{(j k)}=\boldsymbol{\xi}_{j} \times \boldsymbol{\xi}_{k} . &
\end{array}\right.
$$


Note that, in the plane, the cross product of two vectors is a scalar, $\mathbf{u} \times \mathbf{v}=\varepsilon_{i j} u^{i} v^{j} \equiv \overline{\mathbf{u}} J \mathbf{v}$, where $\varepsilon_{i j}$ is the totally antisymmetric symbol, $\varepsilon_{12}=1$. Hence, $\boldsymbol{\xi} \times \boldsymbol{\xi}=\xi^{1} \xi^{2}-\xi^{2} \xi^{1}=2 \xi^{1} \xi^{2}$.

Thus, we now have $N=2 \nu$ ' $\mathcal{Q}$-type' and ' $\mathcal{S}$-type charges, half of them symmetric ( $I$-type), the other half antisymmetric ( $J$-type), but only $\nu$ 'T-type' vector-charges $\boldsymbol{\Xi}_{j}$. Note also that we now have two types of 'fermionic Hamiltonians' namely the symmetric $\mathcal{L}_{j k}$ 's and the antisymmetric $\mathcal{H}_{j k}$ 's.

The super-commutators and super-Poisson brackets, calculated in the usual way, are rather complicated. They are listed in the Appendix. The particular case of $\nu=1$, $\epsilon_{1}=\epsilon_{2}=1$, which plays a significant rôle in Chern-Simons theory, is worked out in Section 8 .

\section{A free spinning particle.}

Let us first consider a free, spin- $\frac{1}{2}$ particle in ordinary space and construct its supersymmetry algebra directly. At the pseudoclassical level [9] including spin amounts to adding an anticommuting 3 -vector, $\zeta=\left(\zeta^{a}\right)$, turned into Pauli matrices upon quantization. In the first part of this Section we work in the quantized setting, i.e. with commutators. The Hamiltonian $\mathcal{H}$ is formally the same as for spin $0, \mathcal{H}=\mathbf{p}^{2} /(2 m)$, and is readily seen to be the square of the conserved supercharge

$$
\mathcal{Q}=\frac{1}{\sqrt{m}} \mathbf{p} \cdot \boldsymbol{\zeta} \quad \text { (helicity) }
$$

The commutator of $\mathcal{Q}$ with boosts, $\mathcal{G}=m \mathbf{r}-\mathbf{p} t$, yields another conserved charge, $\boldsymbol{\Xi}$, which turns out to be proportional to $\zeta$,

$$
\boldsymbol{\Xi}=\sqrt{m} \boldsymbol{\zeta} \quad(\text { spin })
$$

Being the sum of two conserved quantities,

$$
\mathcal{J}^{a b}=r^{a} p^{b}-p^{a} r^{b}-\zeta^{a} \zeta^{b} \quad \text { (total angular momentum) }
$$

is also conserved. The bosonic generators $\mathcal{H}, \mathcal{J}, \mathcal{P}, \mathcal{G}, \mathcal{M}$ (see (6.10) below), and the fermionic generators $\mathcal{Q}$ and $\boldsymbol{\Xi}$ form the 15-dimensional Galilei super-algebra of Gauntlett et al. [5]. Commuting the helicity, $\mathcal{Q}$, with the expansion, $\mathcal{K}$, yields a new charge, namely

$$
\mathcal{S}=\sqrt{m}\left(\mathbf{r}-\frac{\mathbf{p}}{m} t\right) \cdot \boldsymbol{\zeta} \quad \text { (super-expansion). }
$$


Hence we find an 18-dimensional, $N=1$ supersymmetric extension of the Schrödinger algebra [5], whose commutation relations correspond to those of $\widetilde{\operatorname{sch}}(3 / 1)$ in $(4.19)$.

The above result can easily be further extended, using our framework. (From now on, we work again at the pseudo-classical level, i.e. with Grassmann variables). Let us chose $N$ anticommuting $n$-vectors, $\zeta=\left(\boldsymbol{\zeta}_{1}, \ldots, \boldsymbol{\zeta}_{N}\right)$. A free non-relativistic particle with mass $m$ and spin $N / 2$ is described by the action [9]

$$
\mathcal{A}=\int\left\{\mathbf{p} \cdot \dot{\mathbf{r}}+\frac{1}{2} \sum_{j=1}^{N} \overline{\boldsymbol{\zeta}}_{j} \dot{\boldsymbol{\zeta}}_{j}-\frac{\mathbf{p}^{2}}{2 m}\right\} d t
$$

with associated equations of motion $\dot{\mathbf{p}}=0, \dot{\mathbf{r}}=\mathbf{p} / m, \dot{\zeta}=0$. The classical motions are straight lines with $\zeta=$ const. The 'space of motions' is hence globally parametrized by

$$
\mathbf{P}=\frac{\mathbf{p}}{m}, \quad \mathbf{Q}=\mathbf{r}-\frac{\mathbf{p}}{m} t, \quad \boldsymbol{\xi}_{j}=\frac{\boldsymbol{\zeta}_{j}}{\sqrt{m}}
$$

In Souriau's language [8] adapted to this (super)setting, this amounts to working with the 'pre-symplectic' two-form

$$
\sigma=d \overline{\mathbf{p}} \wedge d \mathbf{r}+\frac{1}{2} \sum_{j=1}^{N} d \overline{\boldsymbol{\zeta}}_{j} \wedge d \zeta_{j}-d\left(\frac{\mathbf{p}^{2}}{2 m}\right) \wedge d t
$$

on the 'evolution space'

$$
\mathcal{E}=\left\{\left(\begin{array}{c}
\mathbf{p} \\
\mathbf{r} \\
t \\
\zeta
\end{array}\right) \mid \mathbf{p}, \mathbf{r} \in \mathbf{R}^{n} ; t \in \mathbf{R} ; \zeta=\left(\begin{array}{c}
\boldsymbol{\zeta}_{1} \\
\vdots \\
\boldsymbol{\zeta}_{N}
\end{array}\right), \boldsymbol{\zeta}_{j} \in \mathbf{R}_{1}^{n}\right\}
$$

The 2-form $\sigma$ in Eq. (6.7) is readily seen to project to the space of motions as

$$
m\left(d \overline{\mathbf{P}} \wedge d \mathbf{Q}+\frac{1}{2} \sum_{j=1}^{N} d \overline{\boldsymbol{\xi}}_{j} \wedge d \boldsymbol{\xi}_{j}\right)
$$

i.e. $m$-times the super-symplectic form $\omega$ in Eq. (2.3) with $\epsilon_{j}=1$. According to our general theory, the system admits an $N=N_{+}$-super-Schrödinger symmetry given by Eq. (4.6). The associated conserved quantites are obtained therefore by inserting the parameters 
$\mathbf{P}, \mathbf{Q}$ and $\xi$ from Eq. (6.6) into Eq. (4.10), and multiplying by $m$ to yield

$$
\begin{aligned}
\mathcal{J}^{a b} & =r^{a} p^{b}-p^{a} r^{b}-\sum_{j=1}^{N} \zeta_{j}^{a} \zeta_{j}^{b} & & \text { (angular momentum) } \\
\mathcal{H} & =\frac{\mathbf{p}^{2}}{2 m} & & \text { (energy) } \\
\mathcal{D} & =\mathbf{p} \cdot\left(\mathbf{r}-\frac{\mathbf{p}}{m} t\right) & & \text { (dilatations) } \\
\mathcal{K} & =\frac{1}{2} m\left(\mathbf{r}-\frac{\mathbf{p}}{m} t\right)^{2} & & \text { (expansions) } \\
\mathcal{H}_{i j} & =\boldsymbol{\zeta}_{i} \cdot \boldsymbol{\zeta}_{j} & & \text { (fermionic Hamiltonians) } \\
\mathcal{Q}_{j} & =\frac{1}{\sqrt{m}} \mathbf{p} \cdot \boldsymbol{\zeta}_{j} & & \text { (helicities) } \\
\mathcal{S}_{j} & =\sqrt{m}\left(\mathbf{r}-\frac{\mathbf{p}}{m} t\right) \cdot \boldsymbol{\zeta}_{j} & & \text { (super-expansions) } \\
\mathcal{G} & =m \mathbf{r}-\mathbf{p} t & & \text { (Galilean boosts) } \\
\mathcal{P} & =\mathbf{p} & & \text { (linear momentum) } \\
\mathcal{M} & =m & & \text { (mass) } \\
\boldsymbol{\Xi}_{j} & =\sqrt{m} \boldsymbol{\zeta}_{j} & & \text { (spins) }
\end{aligned}
$$

This generalizes the superalgebra of Gauntlett et al. [5] from $N=1$ to any $N$. (The expression of the angular momentum is consistent with having spin- $\left.\frac{1}{2} N\right)$. Notice that for $N \geq 2$, one also gets an extra bosonic o(N) generated by the $\mathcal{H}_{i j}$ 's - whose conservation is, however, trivial.

If $N \geq 2$, an extra term can be added to the Hamiltonian. For $N=2$, for example, we have two anticommuting Grassmann vectors $\zeta_{j}$, and we can consider the new Hamiltonian

$$
\mathcal{H}_{\text {tot }}=\mathcal{H}_{B}+\mathcal{H}_{F}=\frac{\mathbf{p}^{2}}{2 m}-i \omega\left(\boldsymbol{\zeta}_{+} \cdot \boldsymbol{\zeta}_{-}-\boldsymbol{\zeta}_{-} \cdot \boldsymbol{\zeta}_{+}\right)
$$

with $\omega=$ const. Since the new term commutes with all bosonic variables, the motion in space is undisturbed. However, the $\boldsymbol{\zeta}_{ \pm}=\frac{1}{2}\left(\boldsymbol{\zeta}_{1} \pm i \boldsymbol{\zeta}_{2}\right)$ satisfy rather $\dot{\boldsymbol{\zeta}}_{ \pm}=\mp i \omega \boldsymbol{\zeta}_{ \pm}$, so that $\boldsymbol{\zeta}_{ \pm}(t)=e^{\mp i \omega t} \boldsymbol{\zeta}_{ \pm}(0)$. The projection to the space of motions is hence given as $\mathbf{P}=\mathbf{p} / m, \mathbf{Q}=\mathbf{r}-\mathbf{p} t / m$, supplemented by

$$
\boldsymbol{\xi}_{ \pm}=e^{ \pm i \omega t} \frac{\boldsymbol{\zeta}_{ \pm}}{\sqrt{m}}
$$

Then it is easy to check that one still gets the same symplectic form (6.9). In other words, the space of motions is unchanged by the extra term, and it has therefore the same $\widetilde{\operatorname{sch}}(n / N)$ supersymmetry as for $\omega=0-$ realized in a less trivial way. 
Let us mention that, if the space is two dimensional, $n=2$, we have, in addition to the $I$-type SUSY considered before, also the 'exotic' SUSY described in Section 5, with the usual bosonic generators $\mathcal{H}, \mathcal{D}, \mathcal{K}, \nu(\nu-1) / 2$ 'fermionic Hamiltonians' $\mathcal{H}_{i j}=\boldsymbol{\zeta}_{i} \cdot \boldsymbol{\zeta}_{j}$, but also $\nu(\nu+1) / 2$ symmetric charges $\mathcal{L}_{i j}=\boldsymbol{\zeta}_{i} \times \boldsymbol{\zeta}_{j}$. The angular momentum is simply the scalar $\mathcal{J}=\mathbf{r} \times \mathbf{p}$.

The bosonic generators are supplemented by $\nu I$-type and $\nu J$-type supercharges, as well as $\nu$ spin vectors $\boldsymbol{\Xi}_{j}=\boldsymbol{\zeta}_{j} / \sqrt{m} \in \mathbf{R}_{1}^{2}, j=1, \ldots, \nu$. The structure of this algebra is described, for $\nu=1$, in Section 8 .

\section{The symmetries of the harmonic oscillator.}

Let us first rederive the Schrödinger symmetry for an $n$-dimensional bosonic harmonic oscillator [6] in our framework. Let us indeed consider the oscillator with Hamiltonian $H_{B}=\mathbf{p}^{2} /(2 m)+m \omega^{2} \mathbf{r}^{2} / 2$. The classical trajectories are $\mathbf{r}(t)=\mathbf{A} \cos \omega t+(1 / m \omega) \mathbf{B} \sin \omega t$. The space of motions is therefore $\mathbf{R}^{2 n}$, parametrized by $\mathbf{A}$ and $\mathbf{B}$. In fact, using

$$
\mathbf{A}=\mathbf{r} \cos \omega t-\frac{\mathbf{p}}{m \omega} \sin \omega t \quad \text { and } \quad \mathbf{B}=m \omega \mathbf{r} \sin \omega t+\mathbf{p} \cos \omega t
$$

it is readily verified that the presymplectic 2 -form $\sigma$ of the evolution space $\mathcal{E}=\mathbf{R}^{2 n+1}$ parametrized by the triples $(\mathbf{r}, \mathbf{p}, t)$ projects to the space of motions $\mathcal{E} / \operatorname{ker}(\sigma)$ as the canonical symplectic structure of $\mathbf{R}^{2 n}$,

$$
\sigma=d \overline{\mathbf{p}} \wedge d \mathbf{r}-d H_{B} \wedge d t=d \overline{\mathbf{B}} \wedge d \mathbf{A}=m(d \overline{\mathbf{P}} \wedge d \mathbf{Q})
$$

where

$$
\mathbf{P}=\frac{\mathbf{B}}{m} \quad \text { and } \quad \mathbf{Q}=\mathbf{A}
$$

Thus, the space of motions is the same symplectic vector space as for a free particle — and 
carries therefore a Schrödinger symmetry [6]. The components of the moment map read

$$
\left\{\begin{array}{l}
\mathcal{J}_{a b}=r_{a} p_{b}-r_{b} p_{a} \\
\mathcal{P}=\mathbf{B}=m \omega \mathbf{r} \sin \omega t+\mathbf{p} \cos \omega t \\
\mathcal{G}=m \mathbf{A}=m \mathbf{r} \cos \omega t-\frac{\mathbf{p}}{\omega} \sin \omega t \\
\mathcal{H}=\frac{1}{2 m} \mathbf{B}^{2}=\frac{1}{2}\left(m \omega^{2} \mathbf{r}^{2} \sin ^{2} \omega t+\frac{\mathbf{p}^{2}}{m} \cos ^{2} \omega t+\omega \mathbf{r} \cdot \mathbf{p} \sin 2 \omega t\right) \\
\mathcal{M}=m \\
\mathcal{D}=\mathbf{B} \cdot \mathbf{A}=\frac{1}{2}\left(m \omega \mathbf{r}^{2}-\frac{\mathbf{p}^{2}}{m \omega}\right) \sin 2 \omega t+\mathbf{r} \cdot \mathbf{p} \cos 2 \omega t \\
\mathcal{K}=\frac{1}{2} m \mathbf{A}^{2}=\frac{1}{2} m\left(\mathbf{r}^{2} \cos ^{2} \omega t+\left(\frac{\mathbf{p}}{m \omega}\right)^{2} \sin ^{2} \omega t-\frac{\mathbf{r} \cdot \mathbf{p}}{m \omega} \sin 2 \omega t\right) .
\end{array}\right.
$$

The symmetry generators are combinations of those in Eq. (4.10). For example, a time-translation for the oscillator, $\delta t=\epsilon$, appears, in 'free particle' (i.e. space of motions) language, as a time translation by $\epsilon$, followed by an expansion by $\omega^{2} \epsilon$, etc. In the so-called oscillator representation, they are expressed as

$$
\left\{\begin{array}{l}
J_{a b}=\mathcal{J}_{a b}=r_{a} p_{b}-r_{b} p_{a} \\
H_{B}=\mathcal{H}+\omega^{2} \mathcal{K}=\frac{1}{2}\left(\frac{\mathbf{p}^{2}}{m}+m \omega^{2} \mathbf{r}^{2}\right) \\
C_{ \pm}= \pm i\left(\mathcal{H}-\omega^{2} \mathcal{K} \pm i \omega \mathcal{D}\right)= \pm \frac{i}{2 m} e^{\mp 2 i \omega t}(\mathbf{p} \pm i m \omega \mathbf{r})^{2} \\
\mathbf{P}_{ \pm}= \pm i(\mathcal{P} \pm i \omega \mathcal{G})= \pm i e^{\mp i \omega t}(\mathbf{p} \pm i m \omega \mathbf{r}) \\
M=\mathcal{M}=m
\end{array}\right.
$$

with $a, b=1, \ldots, n$. Here $H_{B}, C_{+}$and $C_{-}$generate an $\operatorname{sl}(2, \mathbf{R})$ algebra; the angular momentum $J$ generates o(n); finally, the $\mathbf{P}_{ \pm}$and $M$ span an $n$-dimensional Heisenberg algebra $\mathrm{h}(\mathrm{n})$.

The $N=2$ supersymmetric oscillator in $n$ space dimensions can be described by adding two anticommuting vectors, $\boldsymbol{\zeta}_{1}$ and $\boldsymbol{\zeta}_{2}$ (or $\boldsymbol{\zeta}_{ \pm}=\frac{1}{2}\left(\boldsymbol{\zeta}_{1} \pm i \boldsymbol{\zeta}_{2}\right)$ ). The action is

$$
\mathcal{A}=\int \mathbf{p} \cdot d \mathbf{r}+\frac{1}{2} \sum_{j=1}^{2} \overline{\boldsymbol{\zeta}}_{j} d \boldsymbol{\zeta}_{j}-\left[\frac{\mathbf{p}^{2}}{2 m}+\frac{m \omega^{2} \mathbf{r}^{2}}{2}-i \omega\left(\boldsymbol{\zeta}_{+} \cdot \boldsymbol{\zeta}_{-}-\boldsymbol{\zeta}_{-} \cdot \boldsymbol{\zeta}_{+}\right)\right] d t
$$


The equations of motions for $\mathbf{p}$ and $\mathbf{r}$ are identical to those without spin, while for $\zeta$ we get $d \zeta_{1} / d t=\omega \zeta_{2}, d \zeta_{2} / d t=-\omega \zeta_{1}$. It follows that the trajectory $\mathbf{r}(t)$ is the same as above, and that $\boldsymbol{\zeta}_{ \pm}(t)=e^{\mp i \omega t} \boldsymbol{\zeta}_{ \pm}(0)$. The evolution space is the same as for a free particle, see (6.8) with $N=2$, while Souriau's two-form reads

$$
\sigma=d \overline{\mathbf{p}} \wedge d \mathbf{r}+\frac{1}{2} \sum_{j=1}^{2} d \overline{\boldsymbol{\zeta}}_{j} \wedge d \boldsymbol{\zeta}_{j}-d\left[\frac{\mathbf{p}^{2}}{2 m}+\frac{m \omega^{2} \mathbf{r}^{2}}{2}-i \omega\left(\boldsymbol{\zeta}_{+} \cdot \boldsymbol{\zeta}_{-}-\boldsymbol{\zeta}_{-} \cdot \boldsymbol{\zeta}_{+}\right)\right] \wedge d t
$$

The coordinates on the space of motions (now super-symplectomorphic to $\mathbf{R}^{2 n / n N}$ ) can therefore be chosen as

$$
\mathbf{P}=\frac{\mathbf{B}}{m}, \quad \mathbf{Q}=\mathbf{A}, \quad \boldsymbol{\xi}_{ \pm}=e^{ \pm i \omega t} \frac{\boldsymbol{\zeta}_{ \pm}}{\sqrt{m}}
$$

It is easy to see that $\sigma$ projects to the space of motions to yield once again $m$-times the (super)symplectic form of Eq. (2.3) with $N=2$ and $\epsilon_{j}=1$. Inserting (7.8) into (4.10) and multiplying by $m$ yields the modified expression of the angular momentum including a spin contribution,

$$
\mathcal{J}^{a b}=r^{a} p^{b}-r^{b} p^{a}-\left(\zeta_{+}^{a} \zeta_{-}^{b}+\zeta_{-}^{a} \zeta_{+}^{b}\right)
$$

and provides us with further conserved quantities. Using

$$
\mathcal{P} \pm i m \omega \mathcal{G}=e^{\mp i \omega t}(\mathbf{p} \pm i m \omega \mathbf{r})
$$

we obtain

$$
\left\{\begin{array}{l}
\mathcal{Q}_{ \pm} \mp i m \omega \mathcal{S}_{ \pm}=(\mathbf{p} \mp i m \omega \mathbf{r}) \cdot \frac{\boldsymbol{\zeta}_{ \pm}}{\sqrt{m}} \\
\mathcal{Q}_{ \pm} \pm i m \omega \mathcal{S}_{ \pm}=e^{\mp 2 i \omega t}(\mathbf{p} \pm i m \omega \mathbf{r}) \cdot \frac{\boldsymbol{\zeta}_{ \pm}}{\sqrt{m}}
\end{array}\right.
$$

i.e. $Q_{ \pm}$and $S_{ \pm}$in Eq. (1.3). Similarly,

$$
H_{F}=-i \omega \mathcal{H}_{12}=-i \omega\left(\boldsymbol{\zeta}_{+} \cdot \boldsymbol{\zeta}_{-}-\boldsymbol{\zeta}_{-} \cdot \boldsymbol{\zeta}_{+}\right),
$$

coincides with the fermionic Hamiltonian in (1.2), and

$$
\boldsymbol{\Xi}_{ \pm}=\sqrt{m} e^{ \pm i \omega t} \boldsymbol{\zeta}_{ \pm}
$$


are the generators $\mathbf{T}_{ \pm}$in Eq. (1.3). We have thus confirmed the $\widetilde{\operatorname{sch}}(n / 2)$ supersymmetry found by Beckers et al. [7].

From Eqs (6.6) and (7.1,3) it follows also, that

$$
\left\{\begin{array}{l}
t_{\text {free }}=\frac{1}{\omega} \tan \left(\omega t_{\mathrm{osc}}\right), \\
\mathbf{r}_{\text {free }}=\frac{\mathbf{r}_{\mathrm{osc}}}{\cos \left(\omega t_{\mathrm{osc}}\right)} \\
\boldsymbol{\zeta}_{\text {free }}^{ \pm}=e^{ \pm i\left(\omega t_{\mathrm{osc}}-\tan \left(\omega t_{\mathrm{osc}}\right)\right)} \boldsymbol{\zeta}_{\mathrm{osc}}^{ \pm}
\end{array}\right.
$$

extends Niederer's correspondence [6] to the case of a free, spin-1 particle with Hamiltonian $H_{\text {tot }}=H_{B}+H_{F}$, and the supersymmetric oscillator.

We just mention, for completeness, that in 2 space dimensions we can also have exotic supersymmetry of Section 5. For $\nu=1$ (just one Grasmann vector $\boldsymbol{\zeta} \in \mathbf{R}_{2}^{1}$ ), one gets the fermionic charges

$$
\begin{aligned}
& (\mathbf{p}+i m \omega \mathbf{r}) \cdot \frac{\boldsymbol{\zeta}}{\sqrt{m}}, \\
& (\mathbf{p}+i m \omega \mathbf{r}) \times \frac{\zeta}{\sqrt{m}}, \\
& e^{2 i \omega t}(\mathbf{p}-i m \omega \mathbf{r}) \cdot \frac{\zeta}{\sqrt{m}} \\
& e^{2 i \omega t}(\mathbf{p}-i m \omega \mathbf{r}) \times \frac{\zeta}{\sqrt{m}} .
\end{aligned}
$$

There is now no ' $\mathcal{H}_{12}$-type' extra bosonic charge. There is, however, an ' $\mathcal{L}_{12}$-type' charge, namely

$$
\mathcal{L}=\frac{e^{2 i \omega t}}{m} \boldsymbol{\zeta} \times \boldsymbol{\zeta}
$$

\section{Chern-Simons-Matter Systems.}

In Ref. [1] Leblanc, Lozano, and Min have constructed a novel, 16-dimensional superconformal extension of the planar Galilei group. Describing their theory goes beyond our scope here; we demonstrate, however, that their superalgebra is precisely our 'exotic' extension described in Section 5. Let us in fact assume that $n=2$, and consider the ' $I J$-type' 
extension with $\nu=1$ and $\epsilon_{1}=\epsilon_{2}=1$. The Grassmann space is hence two-dimensional. The corresponding 'exotic super-Schrödinger algebra' is represented by those matrices

$$
Z=\left(\begin{array}{ccccc}
A+a I & b I & 0 & \mathbf{V} & -\sigma I-\sigma_{*} J \\
c I & A-a I & 0 & \mathbf{W} & -\theta I-\theta_{*} J \\
\frac{1}{2} \overline{\mathbf{W}} & -\frac{1}{2} \overline{\mathbf{V}} & 0 & u & -\frac{1}{2} \overline{\mathbf{\Psi}} \\
0 & 0 & 0 & 0 & 0 \\
\theta I-\theta_{*} J & -\sigma I+\sigma_{*} J & 0 & \boldsymbol{\Psi} & S
\end{array}\right)
$$

where $I=I_{2}, J=J_{2} ; A=\omega J \in \mathrm{o}(2) ; a, b, c, u, r \in \mathbf{R} ; \mathbf{V}, \mathbf{W} \in \mathbf{R}^{2} ; S=r J \in \mathrm{o}(2)$; $\sigma, \sigma_{*}, \theta, \theta_{*} \in \mathbf{R}_{1} ; \boldsymbol{\Psi} \in \mathbf{R}_{1}^{2}$. (Note that there is no $R$ but there is an $S-$ cf. (5.9)). The supercommutators $Z^{\prime \prime}=\left[Z, Z^{\prime}\right]$ are found as

$$
\left\{\begin{aligned}
\omega^{\prime \prime} & =\sigma \theta_{*}^{\prime}+\sigma^{\prime} \theta_{*}-\sigma_{*} \theta^{\prime}-\sigma_{*}^{\prime} \theta \\
a^{\prime \prime} & =b c^{\prime}-b^{\prime} c-\left(\sigma \theta^{\prime}+\sigma^{\prime} \theta+\sigma_{*} \theta_{*}^{\prime}+\sigma_{*}^{\prime} \theta_{*}\right) \\
b^{\prime \prime}= & 2\left(a b^{\prime}-a^{\prime} b\right)+2\left(\sigma \sigma^{\prime}+\sigma_{*} \sigma_{*}^{\prime}\right) \\
c^{\prime \prime}= & 2\left(c a^{\prime}-c^{\prime} a\right)-2\left(\theta \theta^{\prime}+\theta_{*} \theta_{*}^{\prime}\right) \\
\mathbf{V}^{\prime \prime}= & J\left(\omega \mathbf{V}^{\prime}-\omega^{\prime} \mathbf{V}\right)+a \mathbf{V}^{\prime}-a^{\prime} \mathbf{V}+b \mathbf{W}^{\prime}-b^{\prime} \mathbf{W}-\left(\sigma \mathbf{\Psi}^{\prime}+\sigma^{\prime} \mathbf{\Psi}\right) \\
& \quad-J\left(\sigma_{*} \mathbf{\Psi}^{\prime}+\sigma_{*}^{\prime} \mathbf{\Psi}\right) \\
\mathbf{W}^{\prime \prime}= & J\left(\omega \mathbf{W}^{\prime}-\omega^{\prime} \mathbf{W}\right)-a \mathbf{W}^{\prime}+a^{\prime} \mathbf{W}+c \mathbf{V}^{\prime}-c^{\prime} \mathbf{V}-\left(\theta \mathbf{\Psi}^{\prime}+\theta^{\prime} \mathbf{\Psi}\right) \\
& \quad-J\left(\theta_{*} \mathbf{\Psi}^{\prime}+\theta_{*}^{\prime} \mathbf{\Psi}\right) \\
u^{\prime \prime}= & -\overline{\mathbf{V}} \mathbf{W}^{\prime}+\overline{\mathbf{V}^{\prime}} \mathbf{W}-\overline{\mathbf{\Psi}} \boldsymbol{\Psi}^{\prime} \\
r^{\prime \prime}= & 2\left(\sigma \theta_{*}^{\prime}+\sigma^{\prime} \theta_{*}-\theta \sigma_{*}^{\prime}-\theta^{\prime} \sigma_{*}\right)
\end{aligned}\right.
$$

and

$$
\left\{\begin{aligned}
\sigma^{\prime \prime} & =\sigma_{*} \omega^{\prime}-\sigma_{*}^{\prime} \omega+a \sigma^{\prime}-a^{\prime} \sigma+b \theta^{\prime}-b^{\prime} \theta+r \sigma_{*}^{\prime}-r^{\prime} \sigma_{*} \\
\sigma_{*}^{\prime \prime} & =-\sigma \omega^{\prime}+\sigma^{\prime} \omega+a \sigma_{*}^{\prime}-a^{\prime} \sigma_{*}+b \theta_{*}^{\prime}-b^{\prime} \theta_{*}-r \sigma^{\prime}+r^{\prime} \sigma \\
\theta^{\prime \prime} & =-a \theta^{\prime}+a^{\prime} \theta+\theta_{*} \omega^{\prime}-\theta_{*}^{\prime} \omega-\theta_{*} r^{\prime}+\theta_{*}^{\prime} r+c \sigma^{\prime}-c^{\prime} \sigma \\
\theta_{*}^{\prime \prime} & =-a \theta_{*}^{\prime}+a^{\prime} \theta_{*}-\theta \omega^{\prime}+\theta^{\prime} \omega+\theta r^{\prime}-\theta^{\prime} r+c \sigma_{*}^{\prime}-c^{\prime} \sigma_{*} \\
\boldsymbol{\Psi}^{\prime \prime} & =-\sigma \mathbf{W}^{\prime}+\sigma^{\prime} \mathbf{W}+\theta \mathbf{V}^{\prime}-\theta^{\prime} \mathbf{V} \\
& -J\left(\theta_{*} \mathbf{V}^{\prime}-\theta_{*}^{\prime} \mathbf{V}\right)+J\left(\sigma_{*} \mathbf{W}^{\prime}-\sigma_{*}^{\prime} \mathbf{W}\right)+J\left(r \mathbf{\Psi}^{\prime}-r^{\prime} \mathbf{\Psi}\right) .
\end{aligned}\right.
$$


The formula (5.11) of the supermoment map yields now 16 supercharges, namely

$$
\left\{\begin{array}{rlrl}
\mathcal{J} & =\mathbf{Q} \times \mathbf{P}, & & \mathcal{Q}=\mathbf{P} \cdot \boldsymbol{\xi}, \\
\mathcal{H} & =\frac{1}{2}\|\mathbf{P}\|^{2}, & & \mathcal{Q}^{*}=\mathbf{P} \times \boldsymbol{\xi}, \\
\mathcal{D} & =\mathbf{P} \cdot \mathbf{Q}, & & \mathcal{S}=\mathbf{Q} \cdot \boldsymbol{\xi}, \\
\mathcal{K} & =\frac{1}{2}\|\mathbf{Q}\|^{2}, & & \mathcal{S}^{*}=\mathbf{Q} \times \boldsymbol{\xi}, \\
\mathcal{G} & =\mathbf{Q}, & & \mathbf{\Xi}=\boldsymbol{\xi}, \\
\mathcal{P} & =\mathbf{P}, & & \\
\mathcal{M} & =1, & & \\
\mathcal{L} & =\boldsymbol{\xi} \times \boldsymbol{\xi} . &
\end{array}\right.
$$

Observe that there is just one super-translation vector, $\boldsymbol{\Psi}$, and hence a single 'spin' vector $\boldsymbol{\Xi}$, which does not contribute now to the angular momentum: the space rotations do not affect the Grassmann variable $\boldsymbol{\xi}$. Thus $\mathcal{J}$ is merely the orbital angular momentum.

The even Poisson brackets are those of the direct product of the Schrödinger algebra $\operatorname{sch}(2)$ with an extra o(2). Both sets of supercharges $\mathcal{Q}, \mathcal{S}$ and $\mathcal{Q}^{*}, \mathcal{S}^{*}$ extend the bosonic $\operatorname{sl}(2, \mathbf{R})$ generated by $\mathcal{H}, \mathcal{D}, \mathcal{K}$ into $\operatorname{osp}(1 / 1)$ but they fail to close with $\mathcal{L}$ into $\operatorname{osp}(1 / 2)$, because the mixed commutators $\left[\mathcal{Q}, \mathcal{S}^{*}\right]$ and $\left[\mathcal{Q}^{*}, \mathcal{S}\right]$ bring in the angular momentum:

$$
\begin{array}{ll}
{[\mathcal{Q}, \mathcal{S}]=0,} & {\left[\mathcal{Q}^{*}, \mathcal{S}^{*}\right]=0} \\
{\left[\mathcal{Q}, \mathcal{S}^{*}\right]=-\mathcal{J}+\mathcal{L},} & {\left[\mathcal{Q}^{*}, \mathcal{S}\right]=\mathcal{J}-\mathcal{L}}
\end{array}
$$

But $\mathcal{J}$ satisfies now non-trivial commutation relations with the supercharges,

$$
\begin{array}{llrl}
{[\mathcal{J}, \mathcal{Q}]} & =\mathcal{Q}^{*}, & & {\left[\mathcal{J}, \mathcal{Q}^{*}\right]=-\mathcal{Q},} \\
{[\mathcal{J}, \mathcal{S}]=\mathcal{S}^{*},} & {\left[\mathcal{J}, \mathcal{S}^{*}\right]=-\mathcal{S} .}
\end{array}
$$

Thus, defining

$$
\mathcal{Y}=\mathcal{J}-\mathcal{L}=\mathbf{Q} \times \mathbf{P}-\boldsymbol{\xi} \times \boldsymbol{\xi},
$$


the generators $\mathcal{H}, \mathcal{D}, \mathcal{K}, \mathcal{Y}$ and $\mathcal{Q}, \mathcal{Q}^{*}, \mathcal{S}, \mathcal{S}^{*}$ satisfy once more the osp $(1 / 2)$ relations,

$$
\begin{array}{llll}
{[\mathcal{Q}, \mathcal{D}]} & =\mathcal{Q} & {\left[\mathcal{Q}^{*}, \mathcal{D}\right]} & =\mathcal{Q}^{*} \\
{[\mathcal{Q}, \mathcal{K}]} & =\mathcal{S} & {\left[\mathcal{Q}^{*}, \mathcal{K}\right]} & =\mathcal{S}^{*} \\
{[\mathcal{Q}, \mathcal{H}]} & =0, & {\left[\mathcal{Q}^{*}, \mathcal{H}\right]} & =0 \\
{[\mathcal{Q}, \mathcal{Y}]} & =\mathcal{Q}^{*} & {\left[\mathcal{Q}^{*}, \mathcal{Y}\right]=-\mathcal{Q}} \\
{[\mathcal{S}, \mathcal{D}]=-\mathcal{S}} & {\left[\mathcal{S}^{*}, \mathcal{D}\right]=-\mathcal{S}^{*}} \\
{[\mathcal{S}, \mathcal{K}]=0} & {\left[\mathcal{S}^{*}, \mathcal{K}\right]=0} \\
{[\mathcal{S}, \mathcal{H}]=-\mathcal{Q}} & {\left[\mathcal{S}^{*}, \mathcal{H}\right]=-\mathcal{Q}^{*}} \\
{[\mathcal{S}, \mathcal{Y}]=\mathcal{S}^{*}} & {\left[\mathcal{S}^{*}, \mathcal{Y}\right]=-\mathcal{S}} \\
{[\mathcal{Q}, \mathcal{Q}]=-2 \mathcal{H}} & {\left[\mathcal{Q}^{*}, \mathcal{Q}^{*}\right]=-2 \mathcal{H}} \\
{[\mathcal{S}, \mathcal{S}]=-2 \mathcal{K}} & {\left[\mathcal{S}^{*}, \mathcal{S}^{*}\right]=-2 \mathcal{K}} \\
{\left[\mathcal{Q}, \mathcal{Q} \mathcal{Q}^{*}=0\right.} & {\left[\mathcal{S}, \mathcal{S}^{*}\right]=0} \\
{[\mathcal{Q}, \mathcal{S}]} & =-\mathcal{D} & {\left[\mathcal{Q}^{*}, \mathcal{S}^{*}\right]=-\mathcal{D}} \\
{\left[\mathcal{Q}, \mathcal{S}{ }^{*}\right]=-\mathcal{Y}} & {\left[\mathcal{Q}^{*}, \mathcal{S}\right]=\mathcal{Y} .}
\end{array}
$$

On the other hand,

$$
\mathcal{Z}=\mathcal{J}-\frac{1}{2} \mathcal{L}=\mathcal{Q} \times \mathcal{P}-\frac{1}{2} \boldsymbol{\xi} \times \boldsymbol{\xi}
$$

commutes with all generators of $\operatorname{osp}(1 / 2)$, so that the homogeneous part is once more a direct product, $\mathrm{o}(2) \times \operatorname{osp}(1 / 2)$.

The generator $\mathcal{Z}$ plays the rôle of ordinary rotations, and $\mathcal{Y}$ behaves as a fermionic Hamiltonian in the conventional case. Both mix spatial and internal rotations. Since $\mathcal{Z}$ satisfies the same commutation relations with the bosonic generators as $\mathcal{J}$ does in (4.13), $\mathcal{Z}, \mathcal{H}, \mathcal{D}, \mathcal{K}, \mathcal{M}, \mathcal{G}, \mathcal{P}$ form the standard Schrödinger algebra $\widetilde{\operatorname{sch}}(2)$.

The generators $\mathcal{G}, \mathcal{P}$ and $\boldsymbol{\Xi}$ span the super-Heisenberg algebra $\mathrm{h}(2 / 1)$,

$$
\left[\mathcal{G}^{a}, \Xi^{b}\right]=0, \quad\left[\mathcal{P}^{a}, \Xi^{b}\right]=0, \quad\left[\Xi^{a}, \Xi^{b}\right]=-\mathcal{M} \delta^{a b}
$$

The remaining relations are exactly the same as in the 'I-type' case, showing that the structure of our new algebra is

$$
\widetilde{\operatorname{sch}}_{e}(1) \cong(\mathrm{o}(2) \times \operatorname{osp}(1 / 2)) \subseteq \mathrm{h}(2 / 1)
$$

cf. Eq. (4.19). The only difference is that there is now just one super-translation. 
Finally, the commutation relations of the superalgebra of Leblanc et al. [1] are indeed recovered from those (8.5-10) of our exotic super-Schrödinger algebra $\widetilde{\operatorname{sch}}_{e}(1)$ by setting

$\begin{array}{lll}\underline{\mathrm{LLM}} & & \underline{\mathrm{DH}} \\ J & = & \mathcal{J}+\mathcal{M}-\frac{1}{4} \mathcal{L} \\ H & = & \mathcal{H} \\ K & = & \mathcal{K} \\ D & = & \frac{1}{2} \mathcal{D} \\ G_{ \pm} & = & \mp \mathcal{G}_{1}+i \mathcal{G}_{2} \\ P_{ \pm} & = & \mp \mathcal{P}_{1}+i \mathcal{P}_{2} \\ N_{F} & = & -\frac{1}{2} \mathcal{L} \\ N_{B} & = & \mathcal{M}+\frac{1}{2} \mathcal{L} \\ Q_{1} & = & i \Xi^{1}-\Xi^{2} \\ Q_{1}^{*} & = & \Xi^{1}-i \Xi^{2} \\ Q_{2} & = & \frac{1}{2}\left(i \mathcal{Q}-\mathcal{Q}^{*}\right) \\ Q_{2}^{*} & = & \frac{1}{2}\left(\mathcal{Q}-i \mathcal{Q}^{*}\right) \\ F & = & \frac{1}{2}\left(\mathcal{S}+i \mathcal{S}^{*}\right) \\ F^{*} & = & \frac{1}{2}\left(i \mathcal{S}+\mathcal{S}^{*}\right) .\end{array}$

\section{Supersymmetry of the monopole and of the magnetic vortex.}

A few years ago, Jackiw [11] pointed out that a spin-0 particle in a Dirac monopole field has an o $(2,1)$ dynamical symmetry, generated by the spin-0 Hamiltonian, $\widehat{\mathcal{H}}_{0}=\boldsymbol{\pi}^{2} /(2 m)$, by the dilatation and by the expansion,

$$
\widehat{\mathcal{D}}=-2 t \widehat{\mathcal{H}}_{0}+\frac{1}{2}(\boldsymbol{\pi} \cdot \mathbf{r}+\mathbf{r} \cdot \boldsymbol{\pi}) \quad \text { and } \quad \widehat{\mathcal{K}}=t^{2} \widehat{\mathcal{H}}_{0}-t \widehat{\mathcal{D}}+\frac{1}{2} m \mathbf{r}^{2},
$$

where $\boldsymbol{\pi}=\mathbf{p}-e \mathbf{A}$ is associated with a monopole vector potential, $\mathbf{A}$. This result was extended to spin- $\frac{1}{2}$ particles by D'Hoker and Vinet [12] who have shown that for the Pauli Hamiltonian

$$
\widehat{\mathcal{H}}=\frac{1}{2 m}\left(\boldsymbol{\pi}^{2}-e \mathbf{B} \cdot \boldsymbol{\sigma}\right),
$$

not only the conformal generators $\widehat{\mathcal{D}}$ and $\widehat{\mathcal{K}}$, but also the 'fermionic' operators

$$
\widehat{\mathcal{Q}}=\frac{1}{\sqrt{2 m}} \boldsymbol{\pi} \cdot \boldsymbol{\sigma} \quad \text { and } \quad \widehat{\mathcal{S}}=\sqrt{\frac{m}{2}} \mathbf{r} \cdot \boldsymbol{\sigma}-t \widehat{\mathcal{Q}}
$$


are conserved. Thus, adding the total angular momentum, the spin system admits an $\mathrm{o}(3) \times \operatorname{osp}(1 / 1)$ conformal supersymmetry.

Recently, Jackiw [13] found that the o $(2,1)$ symmetry, generated by $\widehat{\mathcal{D}}$ and $\widehat{\mathcal{K}}$ is also present for a magnetic vortex; it combines with the angular momentum and the 'exotic' $N=2$ supersymmetry [14] into an o(2) $\times \operatorname{osp}(1 / 2)$ superalgebra [15].

We first show this in a quantum-mechanical context.

Let us start with a spin- $\frac{1}{2}$ particle in a static magnetic field $\mathbf{B}=B(x, y) \hat{\mathbf{z}}$. Dropping the irrelevant $z$ variable, we work in the plane. The model is described by the Pauli Hamiltonian (9.2) with $B=\operatorname{rot} \mathbf{A} \equiv \epsilon^{i j} \partial_{i} A_{j}$. It is easy to see that $\widehat{\mathcal{H}}$ is a perfect square in two different ways: both operators

$$
\widehat{\mathcal{Q}}=\frac{1}{\sqrt{2 m}} \boldsymbol{\pi} \cdot \boldsymbol{\sigma} \quad \text { and } \quad \widehat{\mathcal{Q}}^{*}=\frac{1}{\sqrt{2 m}} \boldsymbol{\pi} \times \boldsymbol{\sigma}
$$

where $\boldsymbol{\sigma}=\left(\sigma_{1}, \sigma_{2}\right)$, satisfy the anticommutation relations

$$
[\widehat{\mathcal{Q}}, \widehat{\mathcal{Q}}]_{+}=\left[\widehat{\mathcal{Q}}^{*}, \widehat{\mathcal{Q}}^{*}\right]_{+}=2 \widehat{\mathcal{H}}
$$

Thus, for any static, purely magnetic field in the plane, $\widehat{\mathcal{H}}$ is an $N=2$ supersymmetric Hamiltonian [14].

Let us assume henceforth that $B$ is the field of a point-like magnetic vortex directed along the $z$-axis, $B=\Phi \delta(\mathbf{r})$, where $\Phi$ is the total magnetic flux. It is straightforward to check that $\widehat{\mathcal{D}}$ and $\widehat{\mathcal{K}}$ as in Eq. (9.1) generate, along with $\widehat{\mathcal{H}}$, the o $(2,1)$ Lie algebra, to which the angular momentum, $\widehat{\mathcal{J}}=\mathbf{r} \times \boldsymbol{\pi}$, adds an extra o(2). Commuting $\widehat{\mathcal{Q}}$ and $\widehat{\mathcal{Q}}^{*}$ with the expansion, $\widehat{\mathcal{K}}$, yields two more fermionic generators, namely

$$
\left\{\begin{array}{c}
\widehat{\mathcal{S}}=i[\widehat{\mathcal{Q}}, \widehat{\mathcal{K}}]=\sqrt{\frac{m}{2}}\left(\mathbf{r}-\frac{\boldsymbol{\pi}}{m} t\right) \cdot \boldsymbol{\sigma}, \\
\widehat{\mathcal{S}}^{*}=i\left[\widehat{\mathcal{Q}}^{*}, \widehat{\mathcal{K}}\right]=\sqrt{\frac{m}{2}}\left(\mathbf{r}-\frac{\boldsymbol{\pi}}{m} t\right) \times \boldsymbol{\sigma} .
\end{array}\right.
$$

Then the same calculation as in Section 8 shows that $\widehat{\mathcal{Y}}=\mathbf{r} \times \boldsymbol{\pi}+\sigma_{3}, \widehat{\mathcal{H}}, \widehat{\mathcal{D}}, \mathcal{K}$ and $\widehat{\mathcal{Q}}, \widehat{\mathcal{Q}}^{*}, \widehat{\mathcal{S}}, \widehat{\mathcal{S}}^{*}$ span the $\operatorname{osp}(1 / 2)$ superalgebra. Adding also $\widehat{\mathcal{Z}}=\mathbf{r} \times \boldsymbol{\pi}+\frac{1}{2} \sigma_{3}$, which commutes with all generators of $\operatorname{osp}(1 / 2)$, we conclude that the full symmetry superalgebra is the 
direct product $\operatorname{osp}(1 / 2) \times \mathrm{o}(2)$, generated by

$$
\left\{\begin{array}{lll}
\widehat{\mathcal{Y}}=\mathbf{r} \times \boldsymbol{\pi}+\sigma_{3}, & \widehat{\mathcal{Q}}=\frac{1}{\sqrt{2 m}} \boldsymbol{\pi} \cdot \boldsymbol{\sigma}, \\
\widehat{\mathcal{H}}=\frac{1}{2 m}\left(\boldsymbol{\pi}^{2}-e B \sigma_{3}\right), & \widehat{\mathcal{Q}}^{*}=\frac{1}{\sqrt{2 m}} \boldsymbol{\pi} \times \boldsymbol{\sigma}, \\
\widehat{\mathcal{D}}=\frac{1}{2}(\boldsymbol{\pi} \cdot \mathbf{q}+\mathbf{q} \cdot \boldsymbol{\pi})+t \frac{e B}{m} \sigma_{3}, & \widehat{\mathcal{S}}=\sqrt{\frac{m}{2}} \mathbf{q} \cdot \boldsymbol{\sigma}, \\
\widehat{\mathcal{K}}=\frac{1}{2} m \mathbf{q}^{2}, & \widehat{\mathcal{S}}^{*}=\sqrt{\frac{m}{2}} \mathbf{q} \times \boldsymbol{\sigma}, \\
\widehat{\mathcal{Z}}=\mathbf{r} \times \boldsymbol{\pi}+\frac{1}{2} \sigma_{3}, & &
\end{array}\right.
$$

where we have introduced $\mathbf{q} \equiv \mathbf{r}-t \boldsymbol{\pi} / m$.

Now we re-derive these results in our framework.

We shall be concerned with the supersymplectic space $\mathbf{R}^{2 n / n}$, where $n=2,3$ to deal respectively with the case of the magnetic vortex $F=\frac{1}{2} F_{j k} d r^{j} \wedge d r^{k}=\Phi \delta(\mathbf{r}) d x \wedge d y$, and the Dirac monopole $F=\frac{1}{2} \epsilon_{i j k} B^{i} d r^{j} \wedge d r^{k}$ with $\mathbf{B}=g \mathbf{r} / r^{3}$. The minimal coupling prescription amounts to replacing the standard supersymplectic structure of $\mathbf{R}^{2 n / n}$, parametrized by $(\mathbf{r}, \boldsymbol{\pi}, \boldsymbol{\xi})$, see (2.3) by

$$
\omega=d \pi_{j} \wedge d r^{j}+\frac{1}{2} d \xi_{j} \wedge d \xi^{j}+\frac{1}{2} e F_{j k} d r^{j} \wedge d r^{k}
$$

where $e$ is the electric charge of the test particle.

1) The Dirac monopole.

The Pauli Hamiltonian,

$$
\mathcal{H}=\frac{1}{2 m}\left(\boldsymbol{\pi}^{2}+e F_{i j} \xi^{i} \xi^{j}\right),
$$

gives rise to the Hamiltonian vectorfield $X_{\mathcal{H}}\left(\right.$ according to $\left.\imath\left(X_{\mathcal{H}}\right) \omega=-d \mathcal{H}\right)$,

$$
m X_{\mathcal{H}}=\pi^{j} \partial_{r^{j}}-e\left(F_{i j} \pi^{i}+\frac{1}{2} \partial_{j} F_{k \ell} \xi^{k} \xi^{\ell}\right) \partial_{\pi_{j}}-e F_{i j} \xi^{i} \partial_{\xi_{j}} .
$$

Routine calculation using the homogeneity property $r^{j} \partial_{j} F_{k \ell}=-2 F_{k \ell}$, shows furthermore that $\mathcal{H}$ and

$$
\left\{\begin{array}{l}
\mathcal{D}=\boldsymbol{\pi} \cdot \mathbf{r}-2 t \mathcal{H} \quad \text { with } \quad X_{\mathcal{D}}=r^{j} \partial_{r^{j}}-\pi_{j} \partial_{\pi_{j}}-t X_{\mathcal{H}} \\
\mathcal{K}=\frac{1}{2} m \mathbf{r}^{2}-t \mathcal{D}+t^{2} \mathcal{H} \quad \text { with } \quad X_{\mathcal{K}}=-r_{j} \partial_{\pi_{j}}-t X_{\mathcal{D}}+t^{2} X_{\mathcal{H}}
\end{array}\right.
$$


form, under Poisson brackets $\{f, g\}=X_{f} g$, and for each value of $t$, an algebra isomorphic to o $(2,1)$. Moreover, the superfunctions

$$
\mathcal{Q}=\frac{\boldsymbol{\pi} \cdot \boldsymbol{\xi}}{\sqrt{m}} \quad \text { and } \quad \mathcal{S}=\sqrt{m} \mathbf{r} \cdot \boldsymbol{\xi}-t \mathcal{Q}
$$

whose hamiltonian vector fields read

$$
X_{\mathcal{Q}}=\frac{1}{\sqrt{m}}\left(\xi^{j} \partial_{r^{j}}-e F_{i j} \xi^{i} \partial_{\pi_{j}}-\pi^{j} \partial_{\xi^{j}}\right)
$$

and

$$
X_{\mathcal{S}}=-\sqrt{m}\left(\xi_{j} \partial_{\pi_{j}}+r^{j} \partial_{\xi^{j}}\right)-t X_{\mathcal{Q}}
$$

extend the bosonic symmetry algebra into osp(1/1).

The extra rotation generators read

$$
X_{\mathcal{Z}_{j k}}=r_{j} \partial_{r^{k}}-r_{k} \partial_{r^{j}}+\pi_{j} \partial_{\pi^{k}}-\pi_{k} \partial_{\pi^{j}}+\xi_{j} \partial_{\xi^{k}}-\xi_{k} \partial_{\xi^{j}}
$$

with

$$
\mathcal{Z}_{j k}=2 r_{[j} \pi_{k]}-\xi_{j} \xi_{k}
$$

\section{2) The magnetic vortex.}

The vortex case is quite similar: using $x \delta(\mathbf{r})=y \delta(\mathbf{r})=0$ and $\mathbf{r} \cdot \delta^{\prime}(\mathbf{r})=-2 \delta(\mathbf{r})$, it is straightforward to find nine Hamiltonian vector fields $X_{\mathcal{H}}, X_{\mathcal{D}}, X_{\mathcal{K}}, X_{\mathcal{Y}}, X_{\mathcal{Q}}, X_{\mathcal{Q}^{*}}, X_{\mathcal{S}}, X_{\mathcal{S}^{*}}$ and $X_{\mathcal{Z}}$ which leave the symplectic form (9.8) invariant, and to check that their Poisson brackets satisfy the $\mathrm{o}(2) \times \operatorname{osp}(1 / 2)$ supercommutation relations. One readily checks that

$$
\begin{cases}\mathcal{Y}=\mathbf{r} \times \boldsymbol{\pi}-\boldsymbol{\xi} \times \boldsymbol{\xi}, & \mathcal{Q}=\frac{\boldsymbol{\pi} \cdot \boldsymbol{\xi}}{\sqrt{m}}, \\ \mathcal{H}=\frac{1}{2 m}\left(\boldsymbol{\pi}^{2}+e B \boldsymbol{\xi} \times \boldsymbol{\xi}\right), & \mathcal{Q}^{*}=\frac{\boldsymbol{\pi} \times \boldsymbol{\xi}}{\sqrt{m}}, \\ \mathcal{D}=\boldsymbol{\pi} \cdot \mathbf{r}-2 t \mathcal{H}, & \mathcal{S}=\sqrt{m} \mathbf{r} \cdot \boldsymbol{\xi}-t \mathcal{Q}, \\ \mathcal{K}=\frac{1}{2} m \mathbf{r}^{2}-t \mathcal{D}+t^{2} \mathcal{H}, & \mathcal{S}^{*}=\sqrt{m} \mathbf{r} \times \boldsymbol{\xi}-t \mathcal{Q}^{*}, \\ \mathcal{Z}=\mathbf{r} \times \boldsymbol{\pi}-\frac{1}{2} \boldsymbol{\xi} \times \boldsymbol{\xi}, & \end{cases}
$$

actually span the pseudoclassical analog of the operator superalgebra (9.7).

Acknowledgements. We are indebted to Professors J.-G. Demers, M. Leblanc and P. Townsend for helpful correspondence. 


\section{Appendix}

In two configuration-space dimensions, $n=2$, the $I J$-type Ansatz (5.7), yields the following 'exotic' superalgebra (see $(5.10,11)$ ): the even super-Poisson brackets read

$$
\begin{aligned}
{\left[\mathcal{J}, \mathcal{G}^{a}\right] } & =-J_{b}^{a} \mathcal{G}^{b} & {\left[\mathcal{J}, \mathcal{P}^{a}\right] } & =-J_{b}^{a} \mathcal{P}^{b} \\
{\left[\mathcal{J}, \mathcal{Q}_{j}\right] } & =\mathcal{Q}_{j}^{*} & {\left[\mathcal{J}, \mathcal{Q}_{j}^{*}\right] } & =-\mathcal{Q}_{j} \\
{\left[\mathcal{J}, \mathcal{S}_{j}\right] } & =\mathcal{S}_{j}^{*} & {\left[\mathcal{J}, \mathcal{S}_{j}^{*}\right] } & =-\mathcal{S}_{j} \\
{[\mathcal{H}, \mathcal{D}] } & =2 \mathcal{H} & {[\mathcal{H}, \mathcal{K}] } & =\mathcal{D} \\
{\left[\mathcal{H}, \mathcal{G}^{a}\right] } & =\mathcal{P}^{a} & & \\
{\left[\mathcal{H}, \mathcal{S}_{j}\right] } & =\mathcal{Q}_{j} & {\left[\mathcal{H}, \mathcal{S}_{j}^{*}\right] } & =\mathcal{Q}_{j}^{*} \\
{[\mathcal{D}, \mathcal{K}] } & =2 \mathcal{K} & {\left[\mathcal{D}, \mathcal{G}^{a}\right] } & =\mathcal{G}^{a} \\
{\left[\mathcal{D}, \mathcal{P}^{a}\right] } & =-\mathcal{P}^{a} & & \\
{\left[\mathcal{D}, \mathcal{Q}_{j}\right] } & =-\mathcal{Q}_{j} & {\left[\mathcal{D}, \mathcal{Q}_{j}^{*}\right] } & =-\mathcal{Q}_{j}^{*} \\
{\left[\mathcal{D}, \mathcal{S}_{j}\right] } & =\mathcal{S}_{j} & {\left[\mathcal{D}, \mathcal{S}_{j}^{*}\right] } & =\mathcal{S}_{j}^{*} \\
{\left[\mathcal{K}, \mathcal{P}^{a}\right] } & =-\mathcal{G}^{a} & & \\
{\left[\mathcal{K}, \mathcal{Q}_{j}\right] } & =-\mathcal{S}_{j} & {\left[\mathcal{K}, \mathcal{Q}_{j}^{*}\right] } & =-\mathcal{S}_{j}^{*} \\
{\left[\mathcal{G}^{a}, \mathcal{P}_{b}\right] } & =-\mathcal{M}_{b}^{a} & & \\
{\left[\mathcal{G}^{a}, \mathcal{Q}_{j}\right] } & =-\Xi_{j}^{a} & & {\left[\mathcal{G}^{a}, \mathcal{Q}_{j}^{*}\right]=-J_{b}^{a} \Xi_{j}^{b} } \\
{\left[\mathcal{P}^{a}, \mathcal{S}_{j}\right] } & =\Xi_{j}^{a} & {\left[\mathcal{P}^{a}, \mathcal{S}_{j}^{*}\right] } & =J_{b}^{a} \Xi_{j}^{b},
\end{aligned}
$$

$$
\begin{aligned}
{\left[\mathcal{H}_{i j}, \mathcal{H}_{k \ell}\right] } & =\mathcal{H}_{i k} g_{j \ell}-\mathcal{H}_{j k} g_{i \ell}+\mathcal{H}_{j \ell} g_{i k}-\mathcal{H}_{i \ell} g_{j k} \\
{\left[\mathcal{H}_{i j}, \mathcal{L}_{k \ell}\right] } & =-\mathcal{L}_{i k} g_{j \ell}+\mathcal{L}_{j k} g_{i \ell}+\mathcal{L}_{j \ell} g_{i k}-\mathcal{L}_{i \ell} g_{j k}
\end{aligned}
$$

$$
\begin{aligned}
{\left[\mathcal{H}_{j k}, \mathcal{Q}_{\ell}\right] } & =-2 \mathcal{Q}_{[j} g_{k] \ell} & {\left[\mathcal{H}_{j k}, \mathcal{Q}_{\ell}^{*}\right] } & =-2 \mathcal{Q}^{*}{ }_{[j} g_{k] \ell} \\
{\left[\mathcal{H}_{j k}, \mathcal{S}_{\ell}\right] } & =-2 \mathcal{S}_{[j} g_{k] \ell} & {\left[\mathcal{H}_{j k}, \mathcal{S}_{\ell}^{*}\right] } & =-2 \mathcal{S}^{*}{ }_{[j} g_{k] \ell} \\
{\left[\mathcal{H}_{j k}, \Xi_{\ell}^{a}\right] } & =-2 \Xi^{a}{ }_{[j} g_{k] \ell}, & &
\end{aligned}
$$

$$
\begin{array}{rlrl}
{\left[\mathcal{L}_{i j}, \mathcal{L}_{k \ell}\right]} & =\mathcal{H}_{i k} g_{j \ell}+\mathcal{H}_{j k} g_{i \ell}+\mathcal{H}_{j \ell} g_{i k}+\mathcal{H}_{i \ell} g_{j k}, \\
{\left[\mathcal{L}_{j k}, \mathcal{Q}_{\ell}\right]} & =2 \mathcal{Q}^{*}{ }_{(j} g_{k) \ell} & {\left[\mathcal{L}_{j k}, \mathcal{Q}_{\ell}^{*}\right]} & =-2 \mathcal{Q}_{(j} g_{k) \ell} \\
{\left[\mathcal{L}_{j k}, \mathcal{S}_{\ell}\right]} & =2 \mathcal{S}^{*}{ }_{(j} g_{k) \ell} & {\left[\mathcal{L}_{j k}, \mathcal{S}_{\ell}^{*}\right]} & =-2 \mathcal{S}_{(j} g_{k) \ell} \\
{\left[\mathcal{L}_{j k}, \Xi_{\ell}^{a}\right]} & =2 J_{b}^{a} \Xi^{b}{ }_{(j} g_{k) \ell}, &
\end{array}
$$


as for the odd super-Poisson brackets, they retain the form

$$
\begin{array}{rlrl}
{\left[\mathcal{Q}_{j}, \mathcal{Q}_{k}\right]} & =-2 \mathcal{H} g_{j k} & & \\
{\left[\mathcal{Q}_{j}, \mathcal{S}_{k}\right]} & =-\mathcal{D} g_{j k}+\mathcal{H}_{j k} & & {\left[\mathcal{Q}_{j}, \mathcal{S}_{k}^{*}\right]=-\mathcal{J} g_{j k}+\mathcal{L}_{j k}} \\
{\left[\mathcal{Q}_{j}, \Xi_{k}^{a}\right]} & =-\mathcal{P}^{a} g_{j k} & & \\
{\left[\mathcal{Q}_{j}^{*}, \mathcal{Q}_{k}^{*}\right]} & =-2 \mathcal{H} g_{j k} & & \\
{\left[\mathcal{Q}_{j}^{*}, \mathcal{S}_{k}\right]} & =\mathcal{J} g_{j k}-\mathcal{L}_{j k} & {\left[\mathcal{Q}_{j}^{*}, \mathcal{S}_{k}^{*}\right]=-\mathcal{D} g_{j k}+\mathcal{L}_{j k}} \\
{\left[\mathcal{Q}_{j}^{*}, \Xi_{k}^{a}\right]} & =J_{b}^{a} \mathcal{P}^{b} g_{j k} & & \\
{\left[\mathcal{S}_{j}, \mathcal{S}_{k}\right]} & =-2 \mathcal{K} g_{j k} & & {\left[\mathcal{S}_{j}, \Xi_{k}^{a}\right]=-\mathcal{G}^{a} g_{j k}} \\
{\left[\mathcal{S}_{j}^{*}, \mathcal{S}_{k}^{*}\right]} & =-2 \mathcal{K} g_{j k} & {\left[\mathcal{S}_{j}^{*}, \Xi_{k}^{a}\right]=J_{b}^{a} \mathcal{G}^{b} g_{j k}} \\
{\left[\Xi_{j}^{a}, \Xi_{k}^{b}\right]} & =-\mathcal{M} \delta^{a b} g_{j k} . &
\end{array}
$$




\section{References}

1 M. Leblanc, G. Lozano and H. Min, Extended superconformal Galilean symmetry in Chern-Simons matter systems, Ann. Phys. (N.Y.) 219, 328 (1992).

${ }^{2}$ R. Jackiw and So-Young Pi, Classical and quantal nonrelativistic Chern-Simons theory, Phys. Rev. D42, 3500 (1990); G. V. Dunne, R. Jackiw, So-Young Pi and C. A. Trugenberger, Self-dual Chern-Simons solitons and two-dimensional non-linear equations, Phys. Rev. D43, 1332 (1991).

${ }^{3}$ U. Niederer, The maximal kinematical invariance group of the free Schrödinger equation, Helv. Phys. Acta 45, 802 (1972); C. R. Hagen, Scale and conformal transformations in Galilean-covariant field theory, Phys. Rev. D5, 377 (1972); G. Burdet, M. Perrin, Many-body realization of the Schrödinger algebra, Lett. Nuovo Cimento 4, 651 (1972).

${ }^{4}$ C. Duval, G. Burdet, H. P. Künzle and M. Perrin, Bargmann structures and NewtonCartan theory, Phys. Rev. D31, 1841 (1985); W. M. Tulczyjew, An intrinsic formulation of nonrelativistic analytical mechanics and wave mechanics, J. Geom. Phys. 2, No. 3, 93 (1985); V. Husssin and S. Sinzinkayo, Conformal symmetry and constants of motion, J. Math. Phys. 26, 1072 (1985); V. Hussin and M. Jacques, On non-relativistic conformal symmetries and invariant tensor fields, J. Phys. A19, 3471 (1986); M. Omote, S. Kamefuchi, Y. Takahashi, Y. Ohnuki, Galilean symmetries, in Symmetries in Science III, Proc ' 88 Schloss Hofen Meeting, Gruber and Iachello (eds), p. 323 Plenum: N.Y. (1989); M. Henkel, in Finite-size scaling and numerical simulation of statistical systems, Ch. VIII, p. 353, ed. V. Privman, World Sci. (1990); C. Duval, G. Gibbons and P. Horváthy, Celestial mechanics, conformal structures, and gravitational waves, Phys. Rev. D43, 3907 (1991).

${ }^{5}$ J. P. Gauntlett, J. Gomis and P. K. Townsend, Supersymmetry and the physical-phasespace formulation of spinning particles, Phys. Lett. B248, 288 (1990).

${ }^{6} \mathrm{U}$. Niederer, The maximal kinematical invariance group of the harmonic oscillator, Helv. Phys. Acta 46, 192 (1973). 
7 J. Beckers and V. Hussin, Dynamical supersymmetries of the harmonic oscillator, Phys. Lett. A118, 319 (1986); J. Beckers, D. Dehin and V. Hussin, Symmetries and supersymmetries of the quantum harmonic oscillator, J. Phys. A20, 1137 (1987).

8 J-M Souriau, Structure des systèmes dynamiques, Dunod: Paris (1969).

${ }^{9}$ F. A. Berezin and M. S. Marinov, Classical spin and Grassmann algebra, JETP Lett. 21, 321 (1975); Particle spin dynamics as the Grassmann variant of classical mechanics, Ann. Phys. (N.Y.) 104, p. 336, (1977) F. A. Berezin, Introduction to superanalysis, Ed. by A. A. Kirillov, Reidel: Dordrecht (1987); R. Casalbuoni, On the quantization of systems with anti-commuting variables, Il Nuovo Cimento 33A, 115 (1976); The classical mechanics for Bose-Fermi systems, 33A, 389 (1976); A. Barducci, R. Casalbuoni and L. Lusanna, Supersymmetry and the pseudoclassical relativistic electron, 35A, 377 (1976); J. Harnad and J. P. Paré, Kaluza-Klein aproach to the motion of non-Abelian charged particles with spin, Class. Quant. Grav. 8, 1427 (1991).

10 V. G. Kats, Classification of simple Lie superalgebras, Funct. Anal. Appl. 9, 91 (1975); L. Corwin, Y. Ne'eman and S. Sternberg, Graded Lie algebras in mathematics and physics (Bose-Fermi symmetry), Rev. Mod. Phys. 47, p. 573 (1975); B. Kostant, Graded manifolds, graded Lie theory, and prequantization, in Diff. Geom. Meths. in Math. Phys. LNM 570, p. 177, Springer: Berlin (1977); R. Giachetti, R. Ragionieri, and R. Ricci, Symplectic structures on graded manifolds, Journ. Diff. Geom. 16, 247 (1981); A. El Gradechi, On the Supersymplectic Homogeneous Superspace Underlying the osp(1/2) coherent states, Montréal Preprint CRM-1850 (1993).

11 R. Jackiw, Dynamical symmetry of the magnetic monopole, Ann. Phys. (N.Y.) 129, $183(1980)$.

12 E. D'Hoker and L. Vinet, Supersymmetry of the Pauli equation in the presence of a magnetic monopole, Phys. Lett. 137B, 72 (1984); Superspace formulation of the dynamical symmetries of the Dirac magnetic monopole, Lett. Math. Phys. 8, 439 (1984); Dynamical supersymmetry of the magnetic monopole and the $1 / r^{2}$-potential Comm. Math. Phys. 
97, 391 (1985); E. D'Hoker and L. Vinet, Spectrum (super)symmetries of particles in a Coulomb potential, Nucl. Phys. B260, 79 (1985); E. D'Hoker, V. A. Kostelecký and L. Vinet, in Dynamical groups and spectrum generating algebras, A. Bohm, Y. Ne'eman and A. O. Barut (eds), Vol. 1, p. 339; Singapore: World Scientific (1988).

${ }^{13}$ R. Jackiw, Ann. Phys. (N.Y.) 201, 83 (1990).

${ }^{14}$ M. De Crombrugghe and V. Rittenberg, Supersymmetric Quantum Mechanics, Annals of Physics (N.Y.) 151, 99 (1983).

15 C. J. Park, Nucl. Phys. B376, 99 (1992); J.-G. Demers, Mod. Phys. Lett. 8, 827 (1993); C. Duval and P. A. Horvathy, Exotic supersymmetry of the magnetic vortex, Tours Preprint 60/93 (unpublished).

16 A. Pais and V. Rittenberg, Semisimple graded Lie algebras, J. Math. Phys. 16, 2062 (1975); W. Nahm, M. Scheunert, On the sructure of simple pseudo Lie algebras and their invariant bilinear forms, J. Math. Phys. 17, 868 (1976); M. Scheunert, W. Nahm, V. Rittenberg, Irreducible representation of the $\operatorname{osp}(2,1)$ and $\operatorname{spl}(2,1)$ graded Lie algebras, J. Math. Phys. 18, 155 (1977).

17 G. Marmo, G. Morandi, A. Simoni and E. C. G. Sudarshan, Quasi-invariance and central extensions, Phys. Rev. 37, 2196 (1988).

18 R. Puzalowski, Galilean supersymmetry, Acta Phys. Austriaca 50, 45 (1978); J. A. de Azcárraga and D. Ginestar, Nonrelativistic limit of supersymmetric theories, Journ. Math. Phys. 32, 3500 (1991). 